\section{OPEN ACCESS}

Edited by:

Eduardo Daniel Rosas-Blum, Texas Tech University Health Sciences Center, United States

Reviewed by:

Lutfi Abdulfattah Jaber, Independent Researcher, Taibe, Israel

Denease Francis,

Texas Tech University Health Sciences

Center El Paso, United States

Ranjan Bista,

Texas Tech University Health Sciences

Center El Paso, United States

*Correspondence:

Anju Pradhan Sinha apradhandr@gmail.com

tThese authors have contributed equally to this work

Specialty section:

This article was submitted to

Children and Health,

a section of the journal

Frontiers in Pediatrics

Received: 06 April 2020 Accepted: 18 December 2020 Published: 16 February 2021

Citation:

Mithra P, Khatib MN, Sinha AP, Kumar N. Holla R, Unnikrishnan $B$, Vijayamma R, Nair NS, Gaidhane A

and Quazi Zahiruddin S (2021) Interventions for Addressing Anemia Among Children and Adolescents: An

Overview of Systematic Reviews. Front. Pediatr. 8:549549.

doi: 10.3389/fped.2020.549549

\title{
Interventions for Addressing Anemia Among Children and Adolescents: An Overview of Systematic Reviews
}

\begin{abstract}
Prasanna Mithra ${ }^{1 \dagger}$, Mahalaqua Nazli Khatib ${ }^{2 \dagger}$, Anju Pradhan Sinha ${ }^{3 *}$, Nithin Kumar ${ }^{1}$, Ramesh Holla ${ }^{1}$, Bhaskaran Unnikrishnan ${ }^{1}$, Ratheebhai Vijayamma ${ }^{4}$, N. Sreekumaran Nair ${ }^{5}$, Abhay Gaidhane ${ }^{6}$ and Syed Quazi Zahiruddin ${ }^{6}$
\end{abstract}

'Department of Community Medicine, Kasturba Medical College Mangalore, Manipal Academy of Higher Education, Manipal, India, ${ }^{2}$ Division of Evidence Synthesis, Jawaharlal Nehru Medical College, Datta Meghe Institute of Medical Sciences, Wardha, India, ${ }^{3}$ Division of Reproductive, Maternal and Child Health, Indian Council of Medical Research, New Delhi, India, ${ }^{4}$ Manipal Institute of Communication, Manipal Academy of Higher Education, Manipal, India, ${ }^{5}$ Medical Biometrics \& Informatics (Biostatistics), Jawaharlal Institute of Postgraduate Medical Education and Research, Puducherry, India, ${ }^{6}$ Department of Community Medicine, School of Epidemiology and Public Health, Jawaharlal Nehru Medical College, Datta Meghe Institute of Medical Sciences, Wardha, India

Context: Anemia is a public health problem that can lead to growth, cognitive, and motor impairments.

Objective: To collate evidence on interventions for addressing childhood and adolescent anemia.

Methods: In this overview of systematic reviews, we included Cochrane as well as non-Cochrane systematic reviews (SRs) irrespective of language and publication status. Two sets of review authors independently screened articles for eligibility and extracted data from relevant SRs. We present data in a tabular format and summarize results based on outcome reported, age of participants, and type of interventions. We also adopt a "measurement for change" approach to assess the utility of measurement for development of interventions in childhood and adolescent anemia.

Results: Our search yielded 2,601 records of which 31 SRs were found eligible for inclusion. Results were favorable for fortification and supplementation with clear reduction in the risk of anemia and increase in hemoglobin levels across all age groups. Other interventions reported by the SRs were inconclusive and suggest further research.

Conclusions: Current evidence suggests that fortification or supplementation with iron and micronutrients leads to better reduction in the risk of anemia and improvements in hemoglobin levels among children and adolescents. Results of this overview can help decision makers in informing selection of interventions to address childhood and adolescent anemia.

Review Registration: PROSPERO CRD42016053687. 


\section{INTRODUCTION}

Anemia is a condition in which there is either a decrease in the number of red blood corpuscles (RBCs) or a decrease in the total amount of hemoglobin ( $\mathrm{Hb})(1)$. The World Health Organization (WHO) defines childhood anemia as $\mathrm{Hb}$ concentration below 11 grams per deciliter in children between 6 and 59 months, below 11.5 grams per deciliter in children between 5 and 11 years, and below 12 grams per deciliter in children between 12 and 14 years of age (2).

The highest burden of anemia is in low- and middle-income countries (LMICs). Estimates show that almost 90,000 deaths have occurred due to iron deficiency anemia across all age groups (3). India, with $\sim 89$ million children with anemia is the highest contributor of childhood anemia $(4,5)$. Iron deficiency anemia is the most common cause of anemia on a global level. Other causes of childhood anemia include nutritional deficiencies, such as deficiency of folic acid, iron, or vitamin $\mathrm{B}_{12}$; chronic conditions, such as inflammatory disorders; and parasitic infestations (2). Disorders associated with synthesis of hemoglobin, formation of RBCs, or survival of RBCs are the other, less common causes of anemia in children (2). Malaria, HIV, tuberculosis, and helminthic infestations have been reported to lead to substantial burden of anemia, especially in developing countries $(2,6,7)$. The severity of anemia is higher in children under 8 years old as compared with children over 8 years (8). Regardless of the etiology of anemia, impaired physical growth, motor development, and cognitive development have been observed in anemic children $(7,9-12)$.

WHO and the United Nations International Children's Emergency Fund (UNICEF) recommend multipronged public health preventive strategies, such as supplementation of nutrients, fortification of food with nutrients, educational interventions, and prevention and control of parasitic and protozoal infestations (13).

Our comprehensive search strategy reveals many systematic reviews (SRs) that assess the efficacy of different public health interventions for addressing childhood anemia. However, no synthesis of these reviews has been reported in an overview. Hence, the Indian Council of Medical Research (ICMR) task force on childhood and adolescent anemia commissioned this overview in order to collate evidence on the efficacy of various interventions in addressing childhood and adolescent anemia in order to inform programs and a future research agenda. This review could serve as a baseline for clinicians, stakeholders, and decision makers to intervene in childhood and adolescent anemia.

\section{METHODS}

A letter of intent was invited from Indian scientists with experience in evidence synthesis. A review team of experienced researchers was constituted to work on the overview of SRs. The protocol was registered in the PROSPERO prospective register of systematic reviews (Registration number: CRD42016053687) after consultation with national experts (14).

\section{Inclusion Criteria}

Irrespective of language and publication status, we included SRs, evaluating the effect of

1. Any one intervention, or

2. Combination of interventions, such as iron of ferrous sulfate (FS) or zinc $(\mathrm{Zn})$ or multi micro nutrients (MMN) or vitamin supplementation, or

3. Iron or FS or $\mathrm{Zn}$ or MMN or vitamin fortification, or

4. Anti-helminthic treatment

5. Treatment or prevention of malaria, or

6. Treatment of $H$. pylori, or

7. Water, sanitation, and hygiene (WASH) interventions.

We included SRs designed for addressing anemia in children between 6 months and 19 years of age. We included studies regardless of gender, study setting, and severity of anemia. We excluded non-SRs, SRs targeting populations other than children and adolescents, those done on overtly diseased children or adolescents, those providing data on overlapping age groups, and SRs that did not assess the outcomes of interest of this overview. We assessed outcomes, namely anemia, Hb levels, and adverse events.

\section{Search Methods for Identification of Reviews}

We searched the electronic sources listed below between February 8, 2018, and February 10, 2018, and further updated searches between January 5, 2019, and February 7, 2019: Cochrane Database for Systematic Reviews, MEDLINE via PubMed, Database of Abstracts of Reviews of Effects, Cumulative Index to Nursing and Allied Health Literature (CINAHL), Excerpta Medica dataBASE (EMBASE), Campbell Collaboration Online Library of Systematic Reviews (www.campbellcollaboration.org/library.html), Database of Promoting Health Effectiveness Reviews (https://eppi.ioe.ac.uk/ webdatabases4/Intro.aspx?ID=9), and 3ie Database of Systematic Reviews. The review team, in consultation with an information specialist (RV), decided potential key words to be used for this evidence summary. We developed the search strategy for CENTRAL as in Supplementary File 1. In addition, we hand-searched the bibliographies of all eligible reviews, pertinent clinical guidelines, and textbooks to find relevant reviews. We also communicated and requested specialists in this field to provide related unpublished work.

\section{Selection of Reviews}

We undertook screening of SRs using Covidence SR software (15). Two overview authors (PM and RH) independently screened all SRs (retrieved in the search process) on the basis of title and abstract. Two overview authors ( $\mathrm{RH}$ and $\mathrm{NK}$ ) then independently screened the full texts of identified reviews for inclusion. A third reviewer (BU) resolved disagreement among primary reviewers through discussion. We prepared a PRISMA flow diagram to map the inclusion and exclusion of SRs (16). 


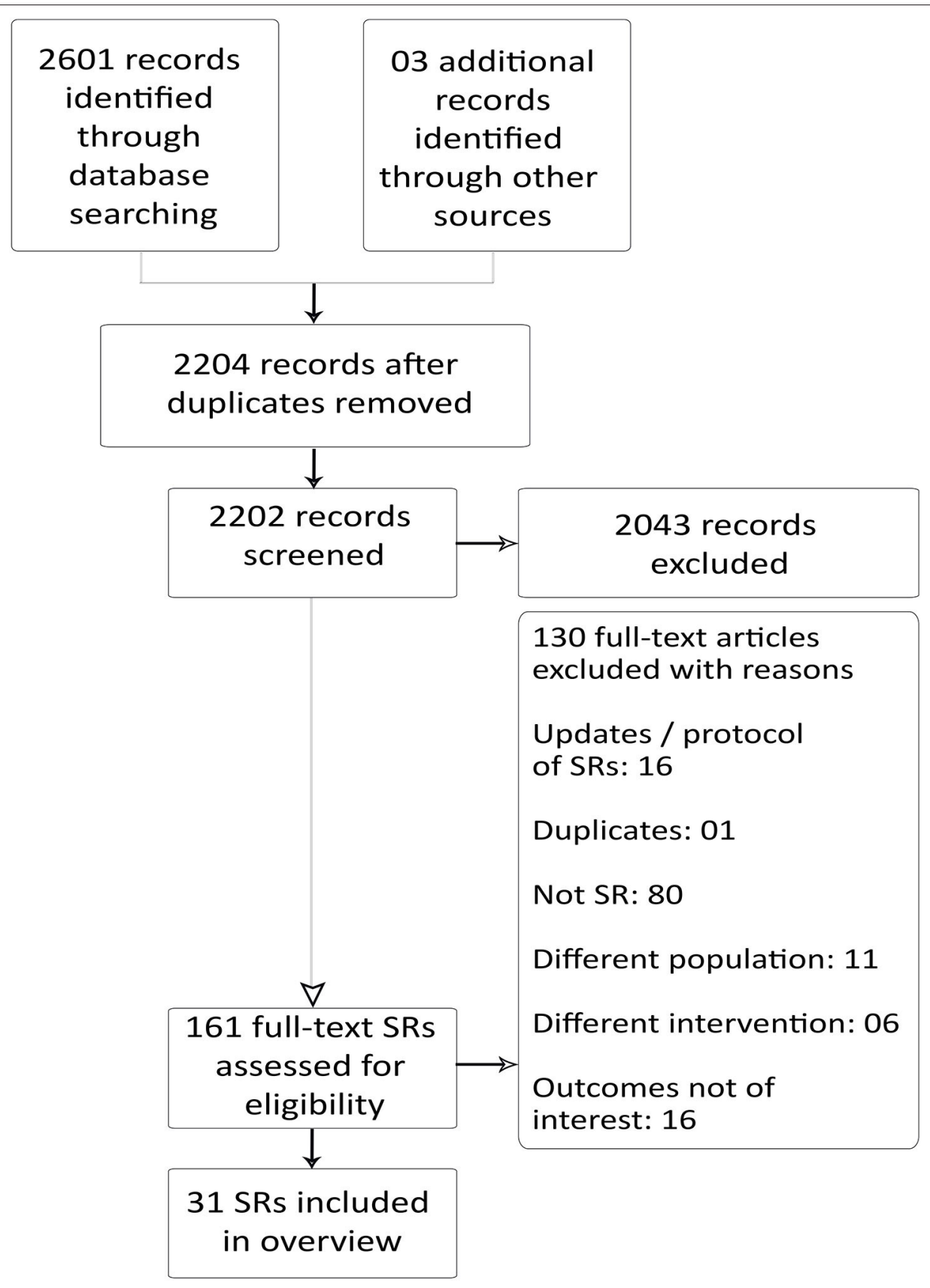

FIGURE 1 | Preferred reporting items for SR and meta-analysis: the PRISMA flow diagram for inclusion and exclusion of studies.

\section{Data Extraction}

We developed and pilot-tested our data extraction form for its suitability and usability. Three review authors (AS, PM, and NK) independently extracted data regarding characteristics of the included participants, interventions, comparisons, outcomes, and methodological quality of the included reviews. We contacted reviewers to seek missing information if any.

\section{Methodological Quality of Included} Reviews Summarized in the Overview

Two review authors (AS and PM) independently evaluated the methodological quality of included reviews with the Revised Assessment of Multiple Systematic Reviews (R-AMSTAR) tool $(17,18)$. 


\section{Data Synthesis}

We followed standard procedures given by the Cochrane Handbook for Systematic Reviews of Interventions (19). We obtained data from included SRs and presented them in a tabular and graphical format. In case of duplicate publications, we considered them as a single report. We summarized results based on outcome reported, age of participants, and type of interventions as risk ratio (RR), mean difference (MD), standardized mean difference (SMD), or weighted mean difference (WMD) with 95\% confidence intervals (95\% CI). As an overview, we mainly focused on summarizing and thematically categorizing the intervention and its effectiveness and not taking a more interpretative approach during synthesis.

\section{RESULTS}

\section{Search Results}

Our search yielded 2,601 records. After removal of duplicates, 2,204 records remained, and they were screened for title and abstract. At this stage, we excluded 2,043 articles, and a total of 161 full-text articles were screened for eligibility. We excluded 130 articles and included 31 SRs that met our inclusion criteria (Figure 1).

\section{Description of Included Reviews}

We included 31 SRs in this overview, the salient features of which are depicted in Table $\mathbf{1}$.

We included nine Cochrane reviews $(20,21,28,31,38$, $41,46,49,50)$ and 22 non-Cochrane SRs $(22-27,29,30,32-$ 37, 39, 40, 42-45, 47, 48). Except for Cembranel et al. (23), all other reviews are in the English language. Updating the version of existing SRs was reported only by Cochrane reviews. The review by Neuberger et al. (21) is the updated version of previous publications $(51,52)$. Taylor-Robinson et al. (46) is updated from a previous five Cochrane reviews (53-55). One Cochrane review by De-Regil et al. (38) has been reprinted in a Cochrane review journal (56), and hence, to avoid duplication, we considered both reviews as a single report (38). Abdullah et al. (27), Best et al. (39), McDonagh et al. (42), and Dangour et al. (49) did not undertake meta-analysis.

Included reviews differed in the age groups of participants: 11 reviews included participants $<5$ years of age $(23,25-27$, $30,33,35,36,38,40,41)$. Low et al. (20), Das et al. (34), and Huang et al. (48) included participants of age groups beyond the inclusion range in our overview, but they reported separate data for children as a subgroup analysis and, hence, were included. Aaron et al. (32) included a trial on pregnant women that was not included in the meta-analysis of anemia. Another review (35) considered included women but did not identify studies on women and, hence, was included in this overview. Except for Low et al. (24), all included reviews included participants of both genders. All SRs except McDonagh et al. (42) included studies from LMICs and developing countries. Sun et al. (45) included specifically the Chinese population. One SR included studies each on children from malaria endemic areas (21), areas endemic for intestinal helminths (46), and areas endemic for anemia (50).
Reviews covered an array of interventions for prevention, control, and/or treatment of anemia in children and adolescents. We found 11 reviews on iron supplementation (20-29, 44). Six reviews were on fortification $(28,30,33,35,39,57)$; five reviews on supplementation with fortification (41-45); two reviews on deworming $(46,47)$; and one review each on $H$. pylori treatment (48), WASH intervention (49), and antimalarial intervention (50).

\section{Effects of Interventions Anemia}

A total of 18 SRs addressed the effect of different interventions on anemia in children and adolescents $(20-25,28,30-36,40,41$, 45, 50) (Table 2 and Figure 2). We did not find any review that assessed the effect of treatment of deworming, H. pylori infection, or WASH intervention on anemia status.

In infants, iron fortification decreased the risk of anemia by $58 \%(\mathrm{RR}=0.42,95 \% \mathrm{CI}=0.24-0.72$; six studies; 1,234 participants; moderate QoE) (34) and MMN fortification by $41 \%$ $(\mathrm{RR}=0.59,95 \% \mathrm{CI}=0.50-0.70$; three studies; 1809 participants; low QoE) (34).

In children under 2 years of age, home fortification, when used as a preventive measure, showed maximum reduction in the risk of anemia by $46 \%(\mathrm{RR}=0.54,95 \% \mathrm{CI}=0.46-0.64 ; 4,331$ participants $6 \mathrm{M}-2 \mathrm{Y})(40)$. However, when used as a treatment measure, it led to an insignificant increase in the risk of anemia by $4 \%(\mathrm{RR}=1.04,95 \% \mathrm{CI}=0.76-1.41 ; 1,263$ participants $6 \mathrm{M}-2 \mathrm{Y})$ (40). In the same age group, iron supplementation significantly reduced the risk of anemia by $39 \%(\mathrm{RR}=0.61,95 \% \mathrm{CI}=$ $0.50-0.74 ; I^{2}=86 \% ; 17$ studies; 4,825 participants $4-23 \mathrm{M}$ ) (25), followed by home fortification with MMP (compared to placebo/no intervention) by $31 \%(\mathrm{RR}=0.69,95 \% \mathrm{CI}=0.60-$ $0.78 ; I^{2}=19 \%$; six studies; 1,447 participants $4-23 \mathrm{M}$; moderate QoE) (38).

Milk fortification significantly reduced the odds of anemia in healthy children between 6 and 47 months old by 64\% (OR = $0.36,95 \% \mathrm{CI}=0.14-0.91)$ (30). Fortification of food with iron and $\mathrm{MMN}\left(\mathrm{RR}=0.43,95 \% \mathrm{CI}=0.26-0.71 ; I^{2}=80.5 \%\right.$; seven studies, 1,927 participants $6 \mathrm{M}-5 \mathrm{Y})(36)$ and intermittent iron supplementation $(\mathrm{RR}=0.43,95 \% \mathrm{CI}=0.23-0.80$; four studies; 658 apparently healthy children $<5$ years) (28) reduced the risk of anemia significantly by $57 \%$, followed by fortification of milk and cereals with iron by $50 \%(\mathrm{RR}=0.50,95 \% \mathrm{CI}=0.33-0.75$; $I^{2}=71.2 \% ; 11$ studies; 3,100 participants $\left.6 \mathrm{M}-5 \mathrm{Y}\right)(36)$, and point-of-use fortification of milk with MMP by $36 \%(\mathrm{RR}=0.64$, $95 \% \mathrm{CI}=0.44-0.93 ; I^{2}=73 \%$; six studies; 1,706 participants $2-$ $5 \mathrm{Y})$ (31). Single-iron fortification of milk ( $\mathrm{RR}=0.76,95 \% \mathrm{CI}$ $=0.45-1.28 ; I^{2}=0 \%$; four studies; 1,173 participants $6 \mathrm{M}-5 \mathrm{Y}$ ) (36), daily doses of FS $\left(\mathrm{RR}=0.73,95 \% \mathrm{CI}=0.49-1.09 ; I^{2}>\right.$ $75 \%$; participants aged $<5 \mathrm{Y})(23)$, and weekly doses of FS (RR $=0.64,95 \% \mathrm{CI}=0.27-1.54 ; I^{2}>75 \%$; participants $\left.<5 \mathrm{Y}\right)(23)$ showed insignificant reductions in the risk of anemia by 36,27 , and $24 \%$, respectively.

In preschool and school-age children, MMN fortification showed a maximum reduction in the risk of anemia by $55 \%$ ( $\mathrm{RR}=0.45,95 \% \mathrm{CI}=0.22-0.89$; five studies; 1,246 participants) (34), followed by iron fortification by $40 \%(\mathrm{RR}=0.60,95 \% \mathrm{CI}$ 
TABLE 1 | Characteristics of the included systematic reviews.

\begin{tabular}{|c|c|c|c|c|c|}
\hline SN & Study ID & $\begin{array}{l}\text { No of } \\
\text { trials/Sample size }\end{array}$ & Participants & Intervention & Outcomes reported \\
\hline \multicolumn{6}{|c|}{ SUPPLEMENTATION } \\
\hline 1. & Low et al. (20) & $\begin{array}{l}\text { 10-50 years age: } \\
67 / 8,506 \\
\text { 12-18 years } \\
\text { age: } 10 / 3,220\end{array}$ & $\begin{array}{l}\text { Menstruating women aged } \\
10-50 \text { years } \\
\text { Subgroup analysis done for } \\
12-18 \text { years }\end{array}$ & $\begin{array}{l}\text { Daily oral iron } \\
\text { supplementation with or } \\
\text { without other vitamins } \\
\text { (folic acid or vitamin C) }\end{array}$ & $\begin{array}{l}\text { Anemia } \\
\text { Hemoglobin } \\
\text { Biomarkers (Ferritin) }\end{array}$ \\
\hline 2. & $\begin{array}{l}\text { Neuberger et al. } \\
(21)\end{array}$ & $35 / 31,955$ & $\begin{array}{l}<18 \text { years children in } \\
\text { malaria-endemic areas } \pm \\
\text { anemia } \pm \text { malaria }\end{array}$ & $\begin{array}{l}\text { Iron, iron + folic acid, and } \\
\text { iron + antimalarial }\end{array}$ & $\begin{array}{l}\text { Anemia } \\
\text { Hemoglobin } \\
\text { Adverse effects }\end{array}$ \\
\hline 3. & $\begin{array}{l}\text { Mayo-Wilson et al. } \\
\text { (22) }\end{array}$ & $80 / 2,05,401$ & $\begin{array}{l}6 \text { months to } 12 \text { years of } \\
\text { age }\end{array}$ & $\begin{array}{l}\text { Orally administered zinc } \\
\text { supplementation and } \\
\text { non-zinc co-interventions } \\
\text { (e.g., vitamin A, additional } \\
\text { iron) }\end{array}$ & $\begin{array}{l}\text { Anemia } \\
\text { Hemoglobin } \\
\text { Adverse effects } \\
\text { Biomarkers (Fe) }\end{array}$ \\
\hline 4. & $\begin{array}{l}\text { Cembranel et al. } \\
\text { (23) }\end{array}$ & 13/1,699 & Children $<5$ years of age & $\begin{array}{l}\text { Weekly/daily } \\
\text { supplementation of iron }\end{array}$ & $\begin{array}{l}\text { Anemia } \\
\text { Hemoglobin }\end{array}$ \\
\hline 5. & Low et al. (24) & $32 / 7,089$ & $\begin{array}{l}\text { Children between } 3.3 \text { and } \\
15 \text { years of age }\end{array}$ & Iron supplementation & $\begin{array}{l}\text { Anemia } \\
\text { Hemoglobin } \\
\text { Adverse effects } \\
\text { Biomarkers (Ferritin) }\end{array}$ \\
\hline 6. & Pasricha et al. (25) & $33 / 42,015$ & 4-23 months of age & Daily iron supplementation & $\begin{array}{l}\text { Anemia } \\
\text { Hemoglobin } \\
\text { Adverse effects } \\
\text { Biomarkers (Ferritin, iron, } \\
\text { transferrin, } \\
\text { erythrocyte protoporphyrin) }\end{array}$ \\
\hline 7. & $\begin{array}{l}\text { Thompson et al. } \\
\text { (26) }\end{array}$ & $15 / 2,154$ & $2-5$ years & $\begin{array}{l}\text { Daily oral iron } \\
\text { supplementation }\end{array}$ & $\begin{array}{l}\text { Hemoglobin } \\
\text { Biomarkers (Ferritin) }\end{array}$ \\
\hline 8. & Abdullah et al. (27) & $2 / 69$ & 6-30 months & $\begin{array}{l}\text { Oral ferrous glycine sulfate } \\
\text { and ferrous sulfate }\end{array}$ & $\begin{array}{l}\text { MA not done due to high } \\
\text { heterogeneity }\end{array}$ \\
\hline 9. & De-Regil et al. (28) & $33 / 13,114$ & $\begin{array}{l}\text { Apparently healthy children } \\
<12 \text { years }\end{array}$ & $\begin{array}{l}\text { Intermittent oral iron } \\
\text { supplementation alone or } \\
\text { in combination with other } \\
\text { vitamins and minerals }\end{array}$ & $\begin{array}{l}\text { Anemia } \\
\text { Hemoglobin } \\
\text { Adverse effects } \\
\text { Biomarkers (Ferritin) }\end{array}$ \\
\hline 10. & $\begin{array}{l}\text { Ramakrishnan } \\
\text { et al. (29) }\end{array}$ & $21 / 4,651$ & 6 month to 18 years & Iron interventions & Hemoglobin \\
\hline \multicolumn{6}{|c|}{ FORTIFICATION } \\
\hline 11. & $\begin{array}{l}\text { Matsuyama et al. } \\
\text { (30) }\end{array}$ & $\begin{array}{l}15 \text { articles based on } \\
12 \text { studies/NR }\end{array}$ & $\begin{array}{l}\text { Healthy children aged 6-47 } \\
\text { months }\end{array}$ & Fortified milk & $\begin{array}{l}\text { Anemia } \\
\text { Hemoglobin } \\
\text { Biomarkers (Serum Ferritin, } \\
\text { serum transferrin } \\
\text { receptor concentration) }\end{array}$ \\
\hline 12. & De-Regil et al. (31) & $\begin{array}{l}13 \text { trials }(17 \\
\text { reports }) / 5,810\end{array}$ & $\begin{array}{l}\text { Preschool and school-age } \\
\text { children from Latin } \\
\text { America, Africa, and Asia }\end{array}$ & $\begin{array}{l}\text { Point-of-use fortification of } \\
\text { foods with MNP }\end{array}$ & $\begin{array}{l}\text { Anemia } \\
\text { Hemoglobin } \\
\text { Adverse effects } \\
\text { Biomarkers (Ferritin) }\end{array}$ \\
\hline 13. & Aaron et al. (32) & 10 & $\begin{array}{l}50525 \text { apparently healthy } \\
\text { children and women of } \\
\text { reproductive age from } \\
\text { LMICs }\end{array}$ & $\begin{array}{l}\text { Non-dairy MMN fortified } \\
\text { beverages }\end{array}$ & Anemia \\
\hline 14. & Das et al. (33) & 11 & $\begin{array}{l}\text { Children and women } \\
\text { (presented separate data } \\
\text { for children) } \\
<11 \text { year old children, } \\
\text { pre-term infants, } \\
\text { malnourished infants, and } \\
\text { school children with } \\
\text { asymptomatic } \\
\text { zinc deficiency) }\end{array}$ & Zinc fortified formula feeds & Hemoglobin \\
\hline
\end{tabular}


TABLE 1 | Continued

\begin{tabular}{|c|c|c|c|c|c|}
\hline SN & Study ID & $\begin{array}{l}\text { No of } \\
\text { trials/Sample size }\end{array}$ & Participants & Intervention & Outcomes reported \\
\hline 15. & Das et al. (34) & $\begin{array}{l}201 \text { (121 on infants } \\
\text { and children) }\end{array}$ & $\begin{array}{l}\text { Children }<18 \text { years of age } \\
\text { and women (presented } \\
\text { separate data for infants } \\
\text { and children) }\end{array}$ & $\begin{array}{l}\text { Vitamin A, iron and } \\
\text { multiple micronutrients } \\
\text { fortified formula food }\end{array}$ & $\begin{array}{l}\text { Anemia } \\
\text { Hemoglobin } \\
\text { Biomarkers (Ferritin) }\end{array}$ \\
\hline 16. & Salam et al. (35) & 17 & $\begin{array}{l}\text { Women and children (6-66 } \\
\text { months of age) (no studies } \\
\text { identified on women) }\end{array}$ & $\begin{array}{l}\text { Point-of-use powders with } \\
\geq 2 \text { micronutrients in their } \\
\text { formulation (Vit A, C, B 11, } \\
\text { D, B complex, Fe, Zinc, } \\
\text { lysine) }\end{array}$ & $\begin{array}{l}\text { Anemia } \\
\text { Hemoglobin } \\
\text { Biomarkers (Ferritin) }\end{array}$ \\
\hline 17. & Eichler et al. (36) & $18 / 5,468$ & 6 months to 5 years & $\begin{array}{l}\text { Micro-nutrient fortified milk } \\
\text { or cereal food }\end{array}$ & $\begin{array}{l}\text { Anemia } \\
\text { Hemoglobin } \\
\text { Biomarkers (Ferritin) }\end{array}$ \\
\hline 18. & Gera et al. (37) & $60 / 20,827$ & $\begin{array}{l}\text { Apparently healthy } \\
\text { individuals, irrespective of } \\
\text { age. Reported separate } \\
\text { data for children on } \mathrm{Hb}\end{array}$ & Iron-fortified foods & Hemoglobin \\
\hline 19. & De-Regil et al. (38) & & & $\begin{array}{l}\text { Home fortification with } \\
\text { multiple micronutrient } \\
\text { powders }\end{array}$ & \\
\hline 20. & Best et al. (39) & $12 / 6,145$ & $\begin{array}{l}\text { Children between } 5.5 \text { and } \\
18 \text { years }\end{array}$ & $\begin{array}{l}\text { Minimum } 3 \text { micronutrients } \\
\text { added to beverages, } \\
\text { foods }\end{array}$ & Not done \\
\hline 21. & Dewey et al. (40) & $16 / 6,113$ & $\begin{array}{l}\text { Children between } 6 \\
\text { months and } 2 \text { years }\end{array}$ & $\begin{array}{l}\text { Sprinkles, Crushable, or } \\
\text { chewable tablets, } \\
\text { Lipid-based nutrient } \\
\text { supplements, soya based } \\
\text { products. }\end{array}$ & $\begin{array}{l}\text { Anemia } \\
\text { Hemoglobin } \\
\text { Adverse events } \\
\text { Biomarkers (Ferritin) }\end{array}$ \\
\hline \multicolumn{6}{|c|}{ SUPPLEMENTATION WITH FORTIFICATION } \\
\hline 22. & $\begin{array}{l}\text { Kristjansson et al. } \\
(41)\end{array}$ & 32 & $\begin{array}{l}3 \text { months to } 5 \text { years } \\
\text { socio-economically } \\
\text { disadvantaged LMIC and } \\
\text { HICs }\end{array}$ & $\begin{array}{l}\text { Macronutrient } \\
\text { supplementation/fortification }\end{array}$ & $\begin{array}{l}\text { Anemia } \\
\text { Hemoglobin }\end{array}$ \\
\hline 23. & $\begin{array}{l}\text { McDonagh et al. } \\
(42)\end{array}$ & $10 / 5,671$ & $\begin{array}{l}\text { Children aged between } 6 \\
\text { and } 24 \text { months from } \\
\text { developed countries }\end{array}$ & $\begin{array}{l}\text { Oral iron supplementation, } \\
\text { iron fortified formula, and } \\
\text { food }\end{array}$ & MA not done \\
\hline 24. & Gera et al. (43) & $30 / 6,464$ & Birth to 15 years & $\begin{array}{l}\text { Iron and multiple } \\
\text { micronutrient } \\
\text { supplementation } \pm \\
\text { fortification }\end{array}$ & Hemoglobin \\
\hline 25. & Gera et al. (44) & $55 / 27,945$ & $<18$ years of age & $\begin{array}{l}\text { Iron supplementation, } \\
\text { formula milk, fortified } \\
\text { cereals }\end{array}$ & Hemoglobin \\
\hline 26. & Sun et al. (45) & $6 / 676$ & $\begin{array}{l}\text { Chinese children between } \\
6 \text { months and } 13 \text { years } \\
\text { with iron deficiency anemia } \\
\text { in }\end{array}$ & Dietary interventions & Anemia \\
\hline \multicolumn{6}{|c|}{ DEWORMING } \\
\hline 27. & $\begin{array}{l}\text { Taylor-Robinson } \\
\text { et al. (46) }\end{array}$ & 45 & $\begin{array}{l}\text { Children aged } \leq 16 \text { years in } \\
\text { areas endemic for intestinal } \\
\text { helminthes }\end{array}$ & Deworming & Hemoglobin \\
\hline 28. & Girum et al. (47) & 8 & $\begin{array}{l}\text { 10,05,239 school children } \\
\text { from Asia and Africa }\end{array}$ & Deworming & Hemoglobin \\
\hline \multicolumn{6}{|c|}{ H. PYLORI TREATMENT } \\
\hline 29. & Huang et al. (48) & $8 / 450$ & $\begin{array}{l}\text { 2-76 years (subgroup } \\
\text { analysis available for } \\
\text { children) }\end{array}$ & H. pylori treatment & $\begin{array}{l}\text { Hemoglobin } \\
\text { Biomarkers (Ferritin) }\end{array}$ \\
\hline
\end{tabular}




\section{TABLE 1 | Continued}

\begin{tabular}{|c|c|c|c|c|c|}
\hline SN & Study ID & $\begin{array}{l}\text { No of } \\
\text { trials/Sample size }\end{array}$ & Participants & Intervention & Outcomes reported \\
\hline \multicolumn{6}{|c|}{ WASH INTERVENTION } \\
\hline 30. & Dangour et al. (49) & $14 / 22,241$ & $<18$ years & WASH intervention & $\begin{array}{l}\text { Hemoglobin (No MA) } \\
\text { Biomarkers (Ferritin) } \\
\text { (No MA) }\end{array}$ \\
\hline \multicolumn{6}{|c|}{ ANTI-MALARIA INTERVENTION } \\
\hline 31. & Athuman et al. (50) & $6 / 3847$ & $\begin{array}{l}\text { Anemic children between } 2 \\
\text { months and } 9 \text { years } \\
\text { residing in endemic areas }\end{array}$ & $\begin{array}{l}\text { Intermittent preventive } \\
\text { antimalarial treatment }\end{array}$ & Anemia \\
\hline
\end{tabular}

$=0.43-0.84 ; 10$ studies; 2,013 participants) (34), point-of-use fortification of food with micro nutrient powders (MNP) by $34 \%$ $\left(\mathrm{RR}=0.66,95 \% \mathrm{CI}=0.49-0.88 ; I^{2}=73 \% ; 10\right.$ studies; 2,448 participants; moderate QoE) (31), and point-of-use fortification of food with iron, vitamin $\mathrm{A}$, and $\mathrm{Zn}$ by $28 \%$ (RR0.72, 95\%CI $=0.65-0.80 ; I^{2}=0 \%$; seven studies; 1,705 participants with malaria) (31). In children with malaria, point-of-use fortification of food with MNP insignificantly reduced the risk of anemia by $43 \%\left(\mathrm{RR}=0.57,95 \% \mathrm{CI}=0.29-1.14 ; I^{2}=83 \%\right.$; four studies; 934 participants with malaria) (31).

In apparently healthy children under 12 years of age, maximum reduction in the risk of anemia is seen with intermittent iron and MMN supplementation by $84 \%$ (RR $=0.16,95 \% \mathrm{CI}=0.06-0.44$; one study; 107 participants $<12$ years) (28), followed by intermittent iron supplementation (iron alone) by $52 \%(\mathrm{RR}=0.48,95 \% \mathrm{CI}=0.31-0.74$; six studies; 1,074 participants $<12$ years). Daily iron/folic acid (FA)/MMN supplementation showed better results as compared with intermittent iron/FA/MMN supplementation (28).

Daily oral iron supplements with or without other vitamins (FA or vitamin C) reduced the risk of anemia in children between 12 and 18 years by $68 \%\left(\mathrm{RR}=0.32,95 \% \mathrm{CI}=0.11-0.93 ; I^{2}\right.$ $=97 \%$; four studies; 2,169 participants) (20), and in children between 3.3 and 15 years reduced the risk of anemia by $50 \%$ $\left(\mathrm{RR}=0.50,95 \% \mathrm{CI}=0.39-0.64 ; I^{2}=85 \%\right.$; seven studies; 1,763 participants) (24). Administration of non-dairy, MMN-fortified beverages in children between 5 and 18 years in LMICs effectively reduced the risk of anemia by $37 \%(\mathrm{RR}=0.63,95 \% \mathrm{CI}=$ $0.54-0.73 ; I^{2}=17 \%$; six studies; 2,995 participants) (32). In malaria-endemic areas, additional supplementation of iron along with antimalarial treatment reduced the risk of anemia by $56 \%$ $\left(\mathrm{RR}=0.44,95 \% \mathrm{CI}=0.28-0.70 ; I^{2}=40 \%\right.$; two studies; 295 participants $<18 \mathrm{Y}$ ), followed by iron and FA supplementation by $51 \%\left(\mathrm{RR}=0.49,95 \% \mathrm{CI}=0.25-0.99 ; I^{2}=49 \%\right.$; three studies; 633 participants $<18 \mathrm{Y}$ ), and iron supplementation by $37 \%(\mathrm{RR}=$ $0.63,95 \% \mathrm{CI}=0.49-0.82 ; I^{2}=97 \% ; 15$ studies; 3,784 participants $<18 \mathrm{Y})(21)$.

\section{Hb Levels}

Twenty-five reviews addressed the effect of different interventions on $\mathrm{Hb}$ levels in children and adolescents (2026, 28-31, 33-38, 40, 41, 43, 44, 46-48, 50) (Table 3 and
Figure 3). We did not find any review that assessed the effect of WASH intervention on $\mathrm{Hb}$ status.

In infants, MMN fortification showed maximum improvement in $\mathrm{Hb}(\mathrm{SMD}=1.05,95 \% \mathrm{CI}=0.48-1.63$; seven studies; 1,508 participants; moderate QoE) (34), followed by iron fortification $(\mathrm{SMD}=0.81,95 \% \mathrm{CI}=0.31-1.31 ; 12$ studies; 1,834 infants; moderate QoE) (34).

In children under 2 years old, daily iron supplementation showed significant improvements in $\mathrm{Hb}(\mathrm{MD}=7.22 \mathrm{~g} / \mathrm{L}, 95 \% \mathrm{CI}$ $=4.87-9.57 ; I^{2}=94 \% ; 26$ studies; 5,479 participants $4 \mathrm{M}-2 \mathrm{Y}$ ) (25), followed by $\mathrm{MMN}$ fortification $(\mathrm{MD}=5.87 \mathrm{~g} / \mathrm{L}, 95 \% \mathrm{CI}$ $=3.25-8.49$, six studies, 1,447 participants $6 \mathrm{M}-2 \mathrm{Y}$ ) (38), and home fortification $(5.06 \mathrm{~g} / \mathrm{L} ; 95 \% \mathrm{CI}=2.29-7.83,22$ studies, 2,449 participants $6 \mathrm{M}-2 \mathrm{Y})(40)$. Home fortification did not show a significant impact on $\mathrm{Hb}$ levels as compared with iron drops $(-0.91 \mathrm{~g} / \mathrm{L} ; 95 \% \mathrm{CI}=-11.96-10.14 ; 1,263$ participants $6 \mathrm{M}-2 \mathrm{Y})$ (40) or iron supplements $(\mathrm{MD}=-2.36 \mathrm{~g} / \mathrm{L} ; 95 \% \mathrm{CI}=-10.30-$ $5.598, I^{2}=78 \%$, two studies, 278 participants $6 \mathrm{M}-2 \mathrm{Y}$ ) (38).

In children under 5 years old, maximum improvement in $\mathrm{Hb}$ levels was seen with $\mathrm{MMN}$ fortification $(\mathrm{MD}=0.87 \mathrm{~g} / \mathrm{dL}, 95 \% \mathrm{CI}$ $=0.57-1.16 ; I^{2}=82 \%$; eight studies; 1,803 participants $6 \mathrm{M}-5 \mathrm{Y}$ ) (36), followed by daily iron supplementation $(\mathrm{MD}=6.97 \mathrm{~g} / \mathrm{L}$, $95 \% \mathrm{CI}=4.21-9.72 ; I^{2}=82 \%$; nine studies; 1,690 participants 2-5Y; high QoE) (26), intermittent iron supplementation (MD $=6.45 \mathrm{~g} / \mathrm{L}, 95 \% \mathrm{CI}=2.36-10.55$; nine studies, 1,254 apparently healthy participants $<5 \mathrm{Y})(28)$, iron fortification of milk and cereals $\left(\mathrm{MD}=0.62 \mathrm{~g} / \mathrm{dL}, 95 \% \mathrm{CI}=0.34-0.89 ; I^{2}=86.2 \%\right.$; 13 studies, 2,274 participants $6 \mathrm{M}-5 \mathrm{Y}$ ) (36), and daily FS supplementation $(\mathrm{MD}=0.56 \mathrm{mg} / \mathrm{dL}, 95 \% \mathrm{CI}=0.31-0.81$; 14 studies; participants $<5 \mathrm{Y}$ ) (23). Insignificant changes were observed with fortified milk $(\mathrm{MD}=5.89 \mathrm{~g} / \mathrm{L}, 95 \% \mathrm{CI}=-0.24-$ 12.02) (30), point-of-use fortification of food with MNP (MD = $2.02 \mathrm{~g} / \mathrm{L}, 95 \% \mathrm{CI}=-0.87$ to 4.92 ) (31), single iron fortification $(\mathrm{MD}=0.20 \mathrm{~g} / \mathrm{dL}, 95 \% \mathrm{CI}=-0.05$ to 0.45$)(36)$, and weekly FS supplementation $(\mathrm{MD}=0.28 \mathrm{mg} / \mathrm{dL} ; 95 \% \mathrm{CI}=-0.22$ to $0.78)(23)$.

The maximum effect on $\mathrm{Hb}$ in preschool and school-age children was seen with iron fortification (SMD $=0.46,95 \% \mathrm{CI}$ $=0.24-0.67 ; 16$ studies; 3,832 participants; moderate QoE) (34) and $\mathrm{MMN}$ fortification $(\mathrm{SMD}=0.45,95 \% \mathrm{CI}=0.12-0.79$; seven studies; 1,543 participants; moderate QoE) (34) followed by point-of-use fortification with $\mathrm{MMN}(\mathrm{MD}=3.37 \mathrm{~g} / \mathrm{L}$, 95\%CI $=0.94-5.80 ; 11$ studies; 2,746 participants; low QoE) 
TABLE 2 | Findings for anemia in included systematic reviews.

\begin{tabular}{|c|c|c|c|c|c|c|}
\hline SR & & $\begin{array}{l}\text { \#Included } \\
\text { studies }\end{array}$ & \#Participants & Comparison & Meta-analysis & $\begin{array}{l}\text { Quality of } \\
\text { evidence }\end{array}$ \\
\hline \multicolumn{7}{|l|}{ INFANTS } \\
\hline Das et al. (34) & MMN fortification & 3 & 1,809 infants & $\begin{array}{l}\text { MMN fortification verses } \\
\text { unfortified foods/ regular } \\
\text { diet }\end{array}$ & $\begin{array}{l}\operatorname{RR}(95 \% \mathrm{Cl})=0.59 \\
(0.50,0.70)\end{array}$ & Low \\
\hline $\begin{array}{l}\text { Matsuyama et al. } \\
\text { (30) }\end{array}$ & Fortification & NR & $\begin{array}{l}\text { Healthy children }<1 \\
\text { year of age }\end{array}$ & $\begin{array}{l}\text { Fortified milk verses } \\
\text { control }\end{array}$ & $\begin{array}{l}\mathrm{OR}(95 \% \mathrm{Cl})=0.46 \\
(0.19,1.12)\end{array}$ & $N R$ \\
\hline \multicolumn{7}{|l|}{$<2$ YEARS } \\
\hline Pasricha et al. (25) & Iron supplementation & 17 & $\begin{array}{l}4,825 \text { children } \\
\text { between } 4 \text { and } 23 \\
\text { months }\end{array}$ & $\begin{array}{l}\text { Iron supplementation } \\
\text { verses control }\end{array}$ & $\begin{array}{l}\mathrm{RR}(95 \% \mathrm{Cl})=0.61 \\
(0.50,0.74) \\
P<0.0001 \\
R^{2}=86 \%\end{array}$ & $N R$ \\
\hline De-Regil et al. (38) & Fortification & 1 & $\begin{array}{l}145 \text { children } 6-23 \\
\text { months }\end{array}$ & $\begin{array}{l}\text { Home fortification with } \\
\text { multiple micronutrient } \\
\text { powders verses iron } \\
\text { supplements }\end{array}$ & $\begin{array}{l}\mathrm{RR}(\mathrm{M}-\mathrm{H}, \mathrm{RE}, 95 \% \mathrm{Cl}) \\
=0.89(0.58-1.39)\end{array}$ & Low \\
\hline Dewey et al. (40) & Fortification & - & $\begin{array}{l}4,331 \text { children } 6 \\
\text { months to } 2 \text { years }\end{array}$ & $\begin{array}{l}\text { Home fortification as } \\
\text { prevention verses control }\end{array}$ & $\begin{array}{l}\operatorname{RR}(95 \% \mathrm{Cl})=0.54 \\
(0.46,0.64)\end{array}$ & NR \\
\hline Dewey et al. (40) & Fortification & - & $\begin{array}{l}1,263 \text { children } 6 \\
\text { months to } 2 \text { years }\end{array}$ & $\begin{array}{l}\text { Home fortification as } \\
\text { treatment verses control }\end{array}$ & $\begin{array}{l}\operatorname{RR}(95 \% \mathrm{Cl})=1.04 \\
(0.76,1.41)\end{array}$ & $N R$ \\
\hline \multicolumn{7}{|l|}{$<5$ YEARS } \\
\hline $\begin{array}{l}\text { Matsuyama et al. } \\
\text { (30) }\end{array}$ & Fortification & $N R$ & $\begin{array}{l}\text { Healthy children aged } \\
6-47 \text { months from all } \\
\text { countries }\end{array}$ & $\begin{array}{l}\text { Fortified milk verses } \\
\text { control }\end{array}$ & $\begin{array}{l}\text { OR }(95 \% \mathrm{Cl})=0.32 \\
(0.15,0.66)\end{array}$ & NR \\
\hline Eichler et al. (36) & Iron fortification & 11 & $\begin{array}{l}3,100 \text { children from } 6 \\
\text { months to } 5 \text { years of } \\
\text { age }\end{array}$ & $\begin{array}{l}\text { Iron fortification of milk } \\
\text { and cereals verses } \\
\text { non-fortified food }\end{array}$ & $\begin{array}{l}\operatorname{RR}(95 \% \mathrm{Cl})=0.50 \\
(0.33,0.75) \\
R^{2}=71.2 \%\end{array}$ & NR \\
\hline De-Regil et al. (31) & MNP fortification & 6 & $\begin{array}{l}1,706 \text { children aged } \\
24-59 \text { months }\end{array}$ & $\begin{array}{l}\text { Point-of-use fortification } \\
\text { of foods with MNP } \\
\text { verses no intervention or } \\
\text { placebo }\end{array}$ & $\begin{array}{l}\mathrm{RR}(\mathrm{M}-\mathrm{H}, \mathrm{RE}, 95 \% \mathrm{Cl}) \\
=0.64(0.44,0.93) \\
P=0.019 \\
I^{2}=73 \%\end{array}$ & $N R$ \\
\hline Eichler et al. (36) & Iron fortification & 4 & $\begin{array}{l}\text { 1,173 Children } \\
\text { between } 6 \text { months } \\
\text { and } 5 \text { years }\end{array}$ & $\begin{array}{l}\text { Iron single-fortification } \\
\text { verses control }\end{array}$ & $\begin{array}{l}\mathrm{RR}(95 \% \mathrm{Cl})=0.76 \\
(0.45-1.28) \\
P=0.533 \\
R^{2}=0 \%\end{array}$ & NR \\
\hline $\begin{array}{l}\text { Kristjansson et al. } \\
(41)\end{array}$ & $\begin{array}{l}\text { Supplementation and } \\
\text { fortification }\end{array}$ & $\begin{array}{l}1 \text { before-after } \\
\text { studies }\end{array}$ & $\begin{array}{l}110 \text { children between } \\
3 \text { months and } 5 \text { years } \\
\text { of age }\end{array}$ & $\begin{array}{l}\text { Supplementary feeding } \\
\text { verses control }\end{array}$ & $\begin{array}{l}\text { OR }(95 \% \mathrm{Cl})=0.58 \\
(0.24,0.75)\end{array}$ & NR \\
\hline $\begin{array}{l}\text { Cembranel et al. } \\
\text { (23) }\end{array}$ & FS supplementation & - & Children $<5$ years & $\begin{array}{l}\text { Daily doses of FS verses } \\
\text { control }\end{array}$ & $\begin{array}{l}\operatorname{RR}(95 \% \mathrm{Cl})=0.73 \\
(0.49,1.09), P= \\
0.13, P^{2}>75 \%\end{array}$ & NR \\
\hline $\begin{array}{l}\text { Cembranel et al. } \\
\text { (23) }\end{array}$ & FS supplementation & - & Children $<5$ years & $\begin{array}{l}\text { Weekly doses of FS } \\
\text { verses control }\end{array}$ & $\begin{array}{l}\operatorname{RR}(95 \% \mathrm{Cl})=0.64 \\
(0.27,1.54), P= \\
0.33, P^{2}>75 \%\end{array}$ & NR \\
\hline
\end{tabular}


TABLE 2 | Continued

\begin{tabular}{|c|c|c|c|c|c|c|}
\hline SR & & $\begin{array}{l}\text { \#Included } \\
\text { studies }\end{array}$ & \#Participants & Comparison & Meta-analysis & $\begin{array}{l}\text { Quality of } \\
\text { evidence }\end{array}$ \\
\hline $\begin{array}{l}\text { Cembranel et al. } \\
\text { (23) }\end{array}$ & FS supplementation & - & Children $<5$ years & $\begin{array}{l}\text { Daily doses of FS verses } \\
\text { weekly doses }\end{array}$ & $\begin{array}{l}\mathrm{RR}(95 \% \mathrm{Cl})=0.70 \\
(0.41,1.19), P=0.09\end{array}$ & NR \\
\hline De-Regil et al. (28) & Iron supplementation & 4 & $\begin{array}{l}658 \text { apparently healthy } \\
\text { children }<5 \text { years }\end{array}$ & $\begin{array}{l}\text { Intermittent iron } \\
\text { supplements verses } \\
\text { placebo }\end{array}$ & $\begin{array}{l}\mathrm{RR}(\mathrm{M}-\mathrm{H}, \mathrm{RE}, 95 \% \\
\mathrm{Cl})=0.43(0.23 \\
0.80)\end{array}$ & NR \\
\hline De-Regil et al. (28) & Iron supplementation & 3 & $\begin{array}{l}770 \text { apparently healthy } \\
\text { children }<5 \text { years }\end{array}$ & $\begin{array}{l}\text { Intermittent iron } \\
\text { supplements verses daily } \\
\text { iron supplements }\end{array}$ & $\begin{array}{l}\mathrm{RR}(\mathrm{M}-\mathrm{H}, \mathrm{RE}, 95 \% \\
\mathrm{Cl})=1.26(1.05 \\
1.51)\end{array}$ & $N R$ \\
\hline \multicolumn{7}{|c|}{ PRE-SCHOOL AND SCHOOL-GOING CHILDREN } \\
\hline Das et al. (34) & MMN Fortification & 5 & $\begin{array}{l}1,246 \text { pre-school and } \\
\text { school-going children }\end{array}$ & $\begin{array}{l}\text { MMN fortification verses } \\
\text { unfortified foods/regular } \\
\text { diet }\end{array}$ & $\begin{array}{l}\mathrm{RR}(95 \% \mathrm{Cl})=0.45 \\
(0.22,0.89)\end{array}$ & Low \\
\hline Das et al. (34) & Iron Fortification & 10 & $\begin{array}{l}\text { 2,013 pre-school and } \\
\text { school-going children }\end{array}$ & $\begin{array}{l}\text { Iron fortification verses } \\
\text { unfortified foods/ regular } \\
\text { diet }\end{array}$ & $\begin{array}{l}\mathrm{RR}(95 \% \mathrm{Cl})=0.60 \\
(0.43,0.84)\end{array}$ & Moderate \\
\hline De-Regil et al. (31) & Fortification & 10 & $\begin{array}{l}\text { 2,448 pre-school and } \\
\text { school-age children }\end{array}$ & $\begin{array}{l}\text { Point-of-use fortification } \\
\text { of foods with MNP } \\
\text { verses no intervention or } \\
\text { placebo }\end{array}$ & $\begin{array}{l}\mathrm{RR}(\mathrm{M}-\mathrm{H}, \mathrm{RE}, 95 \% \mathrm{Cl}) \\
=0.66(0.49-0.88) \\
P=0.004 \\
R^{2}=73 \%\end{array}$ & Moderate \\
\hline De-Regil et al. (31) & Fortification & 7 & $\begin{array}{l}1,705 \text { pre-school and } \\
\text { school-age children } \\
\text { with malaria }\end{array}$ & $\begin{array}{l}\text { Point-of-use fortification } \\
\text { of foods with iron }+ \\
\text { vitamin } A+\text { zinc verses } \\
\text { no intervention or } \\
\text { placebo }\end{array}$ & $\begin{array}{l}\mathrm{RR}(\mathrm{M}-\mathrm{H}, \mathrm{RE}, 95 \% \mathrm{Cl}) \\
=0.72(0.65,0.80) \\
P=0.47 \\
R^{2}=0 \%\end{array}$ & NR \\
\hline De-Regil et al. (31) & Fortification & 4 & $\begin{array}{l}934 \text { pre-school and } \\
\text { school-age children } \\
\text { with malaria }\end{array}$ & $\begin{array}{l}\text { Point-of-use fortification } \\
\text { of foods with MNP } \\
\text { verses no intervention or } \\
\text { placebo }\end{array}$ & $\begin{array}{l}\mathrm{RR}(\mathrm{M}-\mathrm{H}, \mathrm{RE}, 95 \% \mathrm{Cl}) \\
=0.57(0.29,1.14) \\
P=0.0005 \\
R^{2}=83 \%\end{array}$ & $N R$ \\
\hline \multicolumn{7}{|l|}{$<12$ YEARS } \\
\hline De-Regil et al. (28) & Iron Supplementation & 10 & $\begin{array}{l}1,824 \text { apparently } \\
\text { healthy children }<12 \\
\text { years }\end{array}$ & $\begin{array}{l}\text { Intermittent iron } \\
\text { supplements iron alone } \\
\text { or with other nutrients } \\
\text { verses placebo or no } \\
\text { intervention }\end{array}$ & $\begin{array}{l}\mathrm{RR}(\mathrm{M}-\mathrm{H}, \mathrm{RE}, 95 \% \\
\mathrm{Cl})=0.51(0.37 \\
0.72)\end{array}$ & Moderate \\
\hline De-Regil et al. (28) & Iron Supplementation & 6 & $\begin{array}{l}980 \text { apparently healthy } \\
\text { children }<12 \text { years }\end{array}$ & $\begin{array}{l}\text { Intermittent iron } \\
\text { supplements } \pm \text { other } \\
\text { micronutrients verses } \\
\text { daily iron supplements } \pm \\
\text { other micronutrients }\end{array}$ & $\begin{array}{l}\mathrm{RR}(\mathrm{M}-\mathrm{H}, \mathrm{RE}, 95 \% \\
\mathrm{Cl})=1.23(1.04 \\
1.47) \\
P=0.017 \\
P^{2}=0 \%\end{array}$ & Low \\
\hline De-Regil et al. (28) & Iron Supplementation & 5 & $\begin{array}{l}1,456 \text { apparently } \\
\text { healthy children }<12 \\
\text { years }\end{array}$ & $\begin{array}{l}\text { Intermittent iron } \\
\text { supplements ( } 0-3 \\
\text { months duration) verses } \\
\text { placebo or no } \\
\text { intervention }\end{array}$ & $\begin{array}{l}\mathrm{RR}(\mathrm{M}-\mathrm{H}, \mathrm{RE}, 95 \% \\
\mathrm{Cl})=0.63(0.49 \\
0.82)\end{array}$ & NR \\
\hline De-Regil et al. (28) & Iron Supplementation & 5 & $\begin{array}{l}368 \text { apparently healthy } \\
\text { children }<12 \text { years }\end{array}$ & $\begin{array}{l}\text { Intermittent iron } \\
\text { supplements (>3 months } \\
\text { duration) verses placebo } \\
\text { or no intervention }\end{array}$ & $\begin{array}{l}\mathrm{RR}(\mathrm{M}-\mathrm{H}, \mathrm{RE}, 95 \% \\
\mathrm{Cl})=0.37(0.14 \\
1.02)\end{array}$ & $N R$ \\
\hline De-Regil et al. (28) & Iron Supplementation & 6 & $\begin{array}{l}1074 \text { apparently } \\
\text { healthy children }<12 \\
\text { years }\end{array}$ & $\begin{array}{l}\text { Intermittent iron alone } \\
\text { supplements verses } \\
\text { placebo or no } \\
\text { intervention }\end{array}$ & $\begin{array}{l}\mathrm{RR}(\mathrm{M}-\mathrm{H}, \mathrm{RE}, 95 \% \\
\mathrm{Cl})=0.48(0.31 \\
0.74)\end{array}$ & NR \\
\hline De-Regil et al. (28) & Iron Supplementation & 2 & $\begin{array}{l}593 \text { apparently healthy } \\
\text { children }<12 \text { years }\end{array}$ & $\begin{array}{l}\text { Intermittent iron with folic } \\
\text { acid supplements verses } \\
\text { placebo or no } \\
\text { intervention }\end{array}$ & $\begin{array}{l}\mathrm{RR}(\mathrm{M}-\mathrm{H}, \mathrm{RE}, 95 \% \\
\mathrm{Cl})=0.83(0.66 \\
1.03)\end{array}$ & $N R$ \\
\hline De-Regil et al. (28) & Iron Supplementation & 1 & $\begin{array}{l}50 \text { apparently healthy } \\
\text { children }<12 \text { years }\end{array}$ & $\begin{array}{l}\text { Intermittent iron with } \\
\text { vitamin C supplements } \\
\text { verses placebo or no } \\
\text { intervention }\end{array}$ & $\begin{array}{l}\mathrm{RR}(\mathrm{M}-\mathrm{H}, \mathrm{RE}, 95 \% \\
\mathrm{Cl})=0.06(0.00 \\
0.97)\end{array}$ & NR \\
\hline
\end{tabular}


TABLE 2 | Continued

\begin{tabular}{|c|c|c|c|c|c|c|}
\hline SR & & $\begin{array}{l}\text { \#Included } \\
\text { studies }\end{array}$ & \#Participants & Comparison & Meta-analysis & $\begin{array}{l}\text { Quality of } \\
\text { evidence }\end{array}$ \\
\hline De-Regil et al. (28) & Iron Supplementation & 1 & $\begin{array}{l}107 \text { apparently healthy } \\
\text { children }<12 \text { years }\end{array}$ & $\begin{array}{l}\text { Intermittent iron with } \\
\text { MMN supplements } \\
\text { verses placebo or no } \\
\text { intervention }\end{array}$ & $\begin{array}{l}\mathrm{RR}(\mathrm{M}-\mathrm{H}, \mathrm{RE}, 95 \% \\
\mathrm{Cl})=0.16(0.06 \\
0.44)\end{array}$ & NR \\
\hline De-Regil et al. (28) & Iron Supplementation & 2 & $\begin{array}{l}172 \text { apparently healthy } \\
\text { children }<12 \text { years }\end{array}$ & $\begin{array}{l}\text { Intermittent iron } \\
\text { supplements verses daily } \\
\text { iron supplements (0 to } 3 \\
\text { months duration) }\end{array}$ & $\begin{array}{l}\mathrm{RR}(\mathrm{M}-\mathrm{H}, \mathrm{RE}, 95 \% \\
\mathrm{Cl})=1.24(0.55 \\
2.77)\end{array}$ & NR \\
\hline De-Regil et al. (28) & Iron Supplementation & 4 & $\begin{array}{l}808 \text { apparently healthy } \\
\text { children }<12 \text { years }\end{array}$ & $\begin{array}{l}\text { Intermittent iron } \\
\text { supplements verses daily } \\
\text { iron supplements ( }>3 \\
\text { months) }\end{array}$ & $\begin{array}{l}\mathrm{RR}(\mathrm{M}-\mathrm{H}, \mathrm{RE}, 95 \% \\
\mathrm{Cl})=1.23(1.03 \\
1.47)\end{array}$ & NR \\
\hline De-Regil et al. (28) & Iron Supplementation & 4 & $\begin{array}{l}507 \text { apparently healthy } \\
\text { children }<12 \text { years }\end{array}$ & $\begin{array}{l}\text { Intermittent iron } \\
\text { supplements verses daily } \\
\text { iron supplements (Iron } \\
\text { only) }\end{array}$ & $\begin{array}{l}\mathrm{RR}(\mathrm{M}-\mathrm{H}, \mathrm{RE}, 95 \% \\
\mathrm{Cl})=1.17(0.97 \\
1.42)\end{array}$ & $N R$ \\
\hline De-Regil et al. (28) & Iron supplements & 1 & $\begin{array}{l}366 \text { apparently healthy } \\
\text { children }<12 \text { years }\end{array}$ & $\begin{array}{l}\text { Intermittent iron } \\
\text { supplements verses daily } \\
\text { iron supplements (with } \\
\text { folic acid) }\end{array}$ & $\begin{array}{l}\mathrm{RR}(\mathrm{M}-\mathrm{H}, \mathrm{RE}, 95 \% \\
\mathrm{Cl})=1.55(1.02 \\
2.36)\end{array}$ & NR \\
\hline De-Regil et al. (28) & Iron Supplementation & 1 & $\begin{array}{l}107 \text { apparently healthy } \\
\text { children }<12 \text { years }\end{array}$ & $\begin{array}{l}\text { Intermittent iron } \\
\text { supplements verses daily } \\
\text { iron supplements (with } \\
\text { MMN) }\end{array}$ & $\begin{array}{l}\mathrm{RR}(\mathrm{M}-\mathrm{H}, \mathrm{RE}, 95 \% \\
\mathrm{Cl})=1.31(0.31 \\
5.57)\end{array}$ & NR \\
\hline De-Regil et al. (28) & Iron Supplementation & 6 & $\begin{array}{l}\text { 1,166 apparently } \\
\text { healthy children } \\
\text { between } 5 \text { and } 12 \\
\text { years }\end{array}$ & $\begin{array}{l}\text { Intermittent iron } \\
\text { supplements verses } \\
\text { placebo or no } \\
\text { intervention }\end{array}$ & $\begin{array}{l}\mathrm{RR}(\mathrm{M}-\mathrm{H}, \mathrm{RE}, 95 \% \\
\mathrm{Cl})=0.54(0.33 \\
0.90)\end{array}$ & $N R$ \\
\hline De-Regil et al. (28) & Iron Supplementation & 2 & $\begin{array}{l}145 \text { apparently healthy } \\
\text { children between } 5 \\
\text { and } 12 \text { years }\end{array}$ & $\begin{array}{l}\text { Intermittent iron } \\
\text { supplements verses daily } \\
\text { iron supplements }\end{array}$ & $\begin{array}{l}\mathrm{RR}(\mathrm{M}-\mathrm{H}, \mathrm{RE}, 95 \% \\
\mathrm{Cl})=0.95(0.47 \\
1.91)\end{array}$ & NR \\
\hline $\begin{array}{l}\text { Mayo-Wilson et al. } \\
\text { (22) }\end{array}$ & $\begin{array}{l}\text { Zn/Iron } \\
\text { supplementation }\end{array}$ & 3 & $\begin{array}{l}482 \text { children between } \\
6 \text { months and } 12 \\
\text { years of age }\end{array}$ & $\begin{array}{l}\text { Zinc with iron } \\
\text { supplementation verses } \\
\text { zinc alone }\end{array}$ & $\begin{array}{l}\mathrm{RR}(95 \% \mathrm{Cl})=0.78 \\
(0.67-0.92) \\
P=0.54 \\
R^{2}=0 \%\end{array}$ & NR \\
\hline $\begin{array}{l}\text { Mayo-Wilson et al. } \\
\text { (22) (Anemia } \\
\text { prevalence) }\end{array}$ & $\begin{array}{l}\text { Zn/Iron } \\
\text { supplementation }\end{array}$ & 13 & $\begin{array}{l}\text { 4,287 children } \\
\text { between } 6 \text { months } \\
\text { and } 12 \text { years of age }\end{array}$ & $\begin{array}{l}\text { Orally administered zinc } \\
\text { supplementation verses } \\
\text { no zinc supplementation }\end{array}$ & $\begin{array}{l}R R(95 \% \mathrm{Cl})=1.00 \\
(0.95,1.06) \\
P=0.05 \\
R^{2}=37 \%\end{array}$ & NR \\
\hline Athuman et al. (50) & Deworming & 4 & $\begin{array}{l}\text { 2,237 children } \\
\text { between } 2 \text { months } \\
\text { and } 9 \text { years with } \\
\text { anemia in malaria } \\
\text { endemic areas }\end{array}$ & $\begin{array}{l}\text { Intermittent preventive } \pm \\
\text { antimalarial treatment } \\
\text { verses placebo }\end{array}$ & $\begin{array}{l}\mathrm{RR}(95 \% \mathrm{Cl})=0.97 \\
(0.88-1.07) \\
R^{2}=29 \%\end{array}$ & Moderate \\
\hline Sun et al. (45) & Dietary interventions & 6 & $\begin{array}{l}676 \text { Chinese children } \\
\text { with iron deficiency } \\
\text { anemia in }\end{array}$ & $\begin{array}{l}\text { Dietary interventions } \\
\text { verses control }\end{array}$ & $\begin{array}{l}\mathrm{OR}(\mathrm{FE}, 95 \% \mathrm{Cl})= \\
5.03(3.09,8.18) \\
P=0.85 \\
R^{2}=0 \%\end{array}$ & NR \\
\hline \multicolumn{7}{|l|}{$<18$ YEARS } \\
\hline Low et al. (20) & Iron Supplementation & 4 & $\begin{array}{l}2,169 \text { participants } \\
\text { between } 12 \text { and } 18 \\
\text { years }\end{array}$ & $\begin{array}{l}\text { Daily oral iron } \\
\text { supplements } \pm \text { other } \\
\text { vitamins (folic acid or } \\
\text { vitamin C) verses } \\
\text { control/placebo }\end{array}$ & $\begin{array}{l}\mathrm{RR}(\mathrm{M}-\mathrm{H}, \mathrm{RE}, 95 \% \mathrm{Cl}) \\
=0.32(0.11,0.93) \\
P=0.037 \\
R^{2}=97 \%\end{array}$ & NR \\
\hline Low et al. (24) & Iron supplements & 7 & $\begin{array}{l}\text { 1,763 children } \\
\text { between } 3.3 \text { and } 15 \\
\text { years }\end{array}$ & $\begin{array}{l}\text { Daily iron supplements } \\
\text { verses placebo/anti- } \\
\text { helminthics/Zinc/multivitam }\end{array}$ & $\begin{array}{l}\mathrm{RR}(\mathrm{M}-\mathrm{H}, \mathrm{RE}, 95 \% \mathrm{Cl}) \\
=0.50(0.39-0.64) \\
P \leq 0.001 \\
R^{2}=85 \%\end{array}$ & $N R$ \\
\hline
\end{tabular}


TABLE 2 | Continued

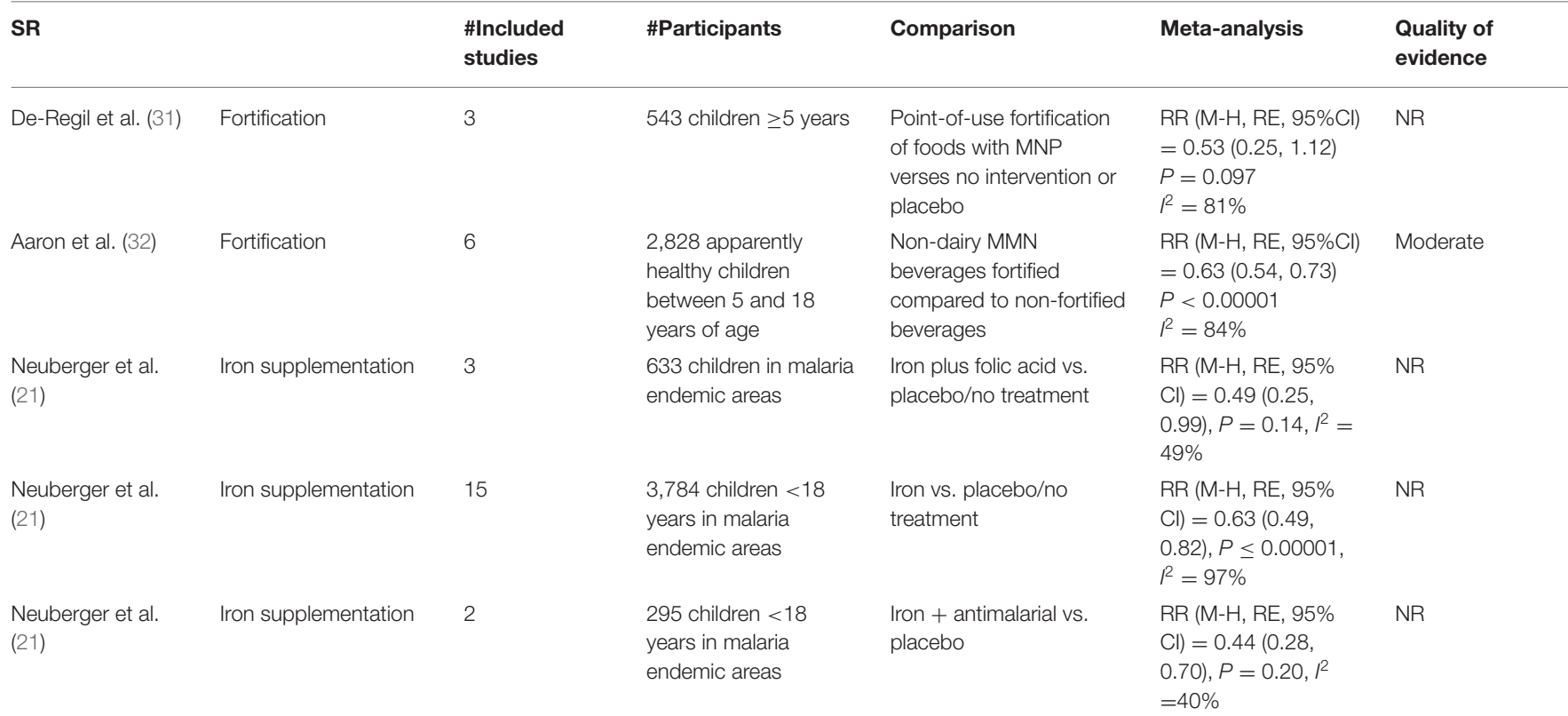

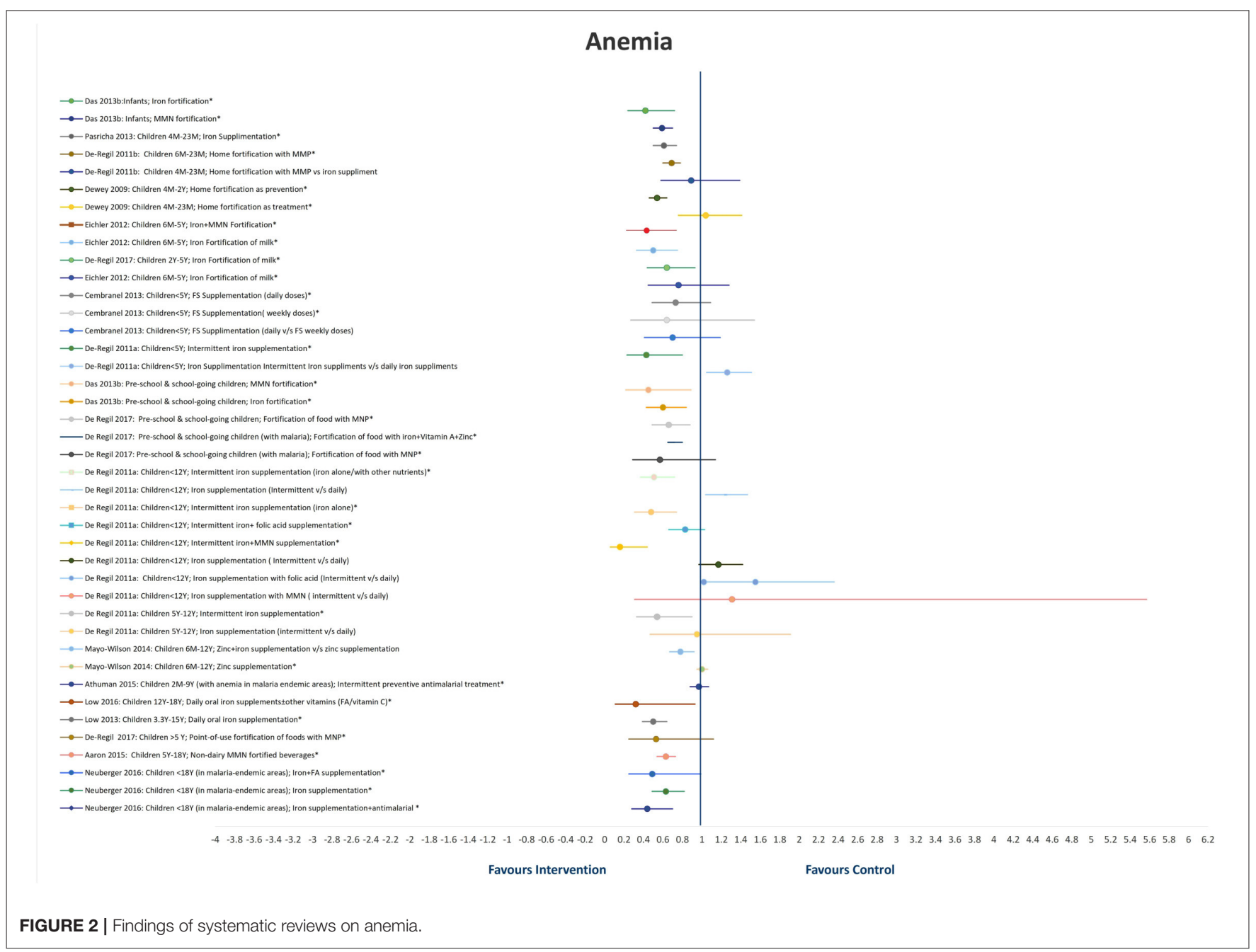


TABLE 3 | Findings for hemoglobin in included systematic reviews.

\begin{tabular}{|c|c|c|c|c|c|c|}
\hline SR & Intervention & $\begin{array}{l}\text { No of } \\
\text { included } \\
\text { studies }\end{array}$ & Number of participants & Comparison & Meta-analysis & $\begin{array}{l}\text { Quality of } \\
\text { evidence }\end{array}$ \\
\hline \multicolumn{7}{|l|}{ INFANTS } \\
\hline Das et al. (34) & MMN fortification & 7 & 1,508 infants & $\begin{array}{l}\text { MMN fortification verses } \\
\text { unfortified foods/regular diet }\end{array}$ & $\begin{array}{l}\operatorname{SMD}(95 \% \mathrm{Cl})=1.05 \\
(0.48,1.63)\end{array}$ & Moderate \\
\hline Das et al. (34) & Iron fortification & 12 & 1,834 infants & $\begin{array}{l}\text { Iron fortification verses } \\
\text { unfortified foods/ regular diet }\end{array}$ & $\begin{array}{l}\operatorname{SMD}(95 \% \mathrm{Cl})=0.81 \\
(0.31,1.31)\end{array}$ & Moderate \\
\hline Gera et al. (37) & Iron fortification & 9 & Infants & $\begin{array}{l}\text { Iron fortification verses } \\
\text { non-fortified food }\end{array}$ & $\begin{array}{l}\text { WMD }(95 \% \mathrm{Cl})=0.5 \\
(0.28,0.72) \mathrm{g} / \mathrm{dL} \\
P \leq 0.001 \\
R^{2}=74.7 \%\end{array}$ & $N R$ \\
\hline Salam et al. (35) & $\begin{array}{l}\text { MNP } \\
\text { supplementation/for }\end{array}$ & $\begin{array}{l}14 \\
\text { ification }\end{array}$ & $\begin{array}{l}8,354 \text { children between } 2 \\
\text { months and } 1 \text { year of age } \\
\text { from developing countries }\end{array}$ & $\begin{array}{l}\text { MNP vs. no intervention or } \\
\text { control }\end{array}$ & $\begin{array}{l}\text { SMD }(\mathrm{IV}, \mathrm{RE}, 95 \% \mathrm{Cl})= \\
0.98(0.55,1.40) \\
P<0.00001 \\
R^{2}=99 \%\end{array}$ & Moderate \\
\hline Das et al. (33) & Zinc fortification & 3 & $\begin{array}{l}92 \text { children (malnourished, } \\
\text { preterm infants, or }<9 \\
\text { years of age) }\end{array}$ & $\begin{array}{l}\text { Zinc fortification verses } \\
\text { control }\end{array}$ & $\begin{array}{l}\text { SMD }(\mathrm{FE}, 95 \% \mathrm{Cl})= \\
-0.11(-0.52,0.31) \\
P=0.45 \\
R^{2}=0 \%\end{array}$ & NR \\
\hline \multicolumn{7}{|c|}{ CHILDREN $<2$ YEARS OF AGE } \\
\hline Pasricha et al. (25) & $\begin{array}{l}\text { Iron } \\
\text { supplementation }\end{array}$ & 26 & $\begin{array}{l}5,479 \text { children between } 4 \\
\text { months and } 2 \text { years of age }\end{array}$ & $\begin{array}{l}\text { Iron supplementation verses } \\
\text { control }\end{array}$ & $\begin{array}{l}\mathrm{MD}(95 \% \mathrm{Cl})=7.22 \\
(4.87-9.57) \mathrm{g} / \mathrm{L} \\
P<0.0001 \\
R^{2}=94 \%\end{array}$ & NR \\
\hline De-Regil et al. (38) & Home fortification & 6 & $\begin{array}{l}\text { 1,447 children between } 6 \\
\text { and } 23 \text { months }\end{array}$ & $\begin{array}{l}\text { Home fortification with MMP } \\
\text { verses placebo/no } \\
\text { intervention }\end{array}$ & $\begin{array}{l}\mathrm{MD}(\mathrm{IV}, \mathrm{RE}, 95 \% \mathrm{Cl})= \\
5.87(3.25-8.49) \mathrm{g} / \mathrm{L}\end{array}$ & Moderate \\
\hline Dewey et al. (40) & Home fortification & 22 & $\begin{array}{l}2,449 \text { between children } 6 \\
\text { months and } 2 \text { years of age }\end{array}$ & $\begin{array}{l}\text { Home fortification as } \\
\text { prevention verses control }\end{array}$ & $5.06(2.29,7.83) \mathrm{g} / \mathrm{L}$ & $N R$ \\
\hline $\begin{array}{l}\text { Gera et al. (44) } \\
\text { (change in } \mathrm{Hb} \text { ) }\end{array}$ & $\begin{array}{l}\text { Iron } \\
\text { supplementation/ } \\
\text { fortification }\end{array}$ & 29 & Children $\leq 2$ years of age & $\begin{array}{l}\text { Iron supplementation/ } \\
\text { fortification verses control }\end{array}$ & $\begin{array}{l}\text { WMD }(\mathrm{RE}, 95 \% \mathrm{Cl})= \\
0.56(0.36,0.76) \mathrm{g} / \mathrm{dL} \\
P<0.001\end{array}$ & NR \\
\hline Gera et al. (37) & Iron fortification & 45 & Children $>1$ year of age & $\begin{array}{l}\text { Iron fortification verses } \\
\text { non-fortified food }\end{array}$ & $\begin{array}{l}\text { WMD }(\mathrm{RE}, 95 \% \mathrm{Cl})= \\
0.49(0.31,0.67) \mathrm{g} / \mathrm{dL} \\
P<0.001 l^{2}=94 \%\end{array}$ & $N R$ \\
\hline Dewey et al. (40) & Home fortification & - & $\begin{array}{l}\text { 1,263 children between } 6 \\
\text { months and } 2 \text { years of age }\end{array}$ & $\begin{array}{l}\text { Home fortification as } \\
\text { treatment verses iron drops }\end{array}$ & $\begin{array}{l}-0.91(-11.96,10.14) \\
g / L\end{array}$ & $N R$ \\
\hline De-Regil et al. (38) & Home fortification & 2 & 278 children $6-23$ months & $\begin{array}{l}\text { Home fortification with MMP } \\
\text { verses iron supplements }\end{array}$ & $\begin{array}{l}\mathrm{MD}(\mathrm{IV}, \mathrm{RE}, 95 \% \mathrm{Cl})= \\
-2.36(-10.30 \text { to } \\
5.59) \mathrm{g} / \mathrm{L} \\
R^{2}=78 \%\end{array}$ & Low \\
\hline \multicolumn{7}{|l|}{$<5$ YEARS } \\
\hline $\begin{array}{l}\text { Thompson et al. } \\
\text { (26) (end point) }\end{array}$ & $\begin{array}{l}\text { Iron } \\
\text { supplementation }\end{array}$ & 9 & $\begin{array}{l}\text { 1,690 children between } 2 \\
\text { and } 5 \text { years of age }\end{array}$ & $\begin{array}{l}\text { Daily iron supplementation } \\
\geq 5 \text { days/week verses control }\end{array}$ & $\begin{array}{l}\mathrm{MD}(95 \% \mathrm{Cl})=6.97 \\
(4.21,9.72) \mathrm{g} / \mathrm{L} \\
P<0.00001 \\
R^{2}=82 \%\end{array}$ & High \\
\hline De-Regil et al. (28) & $\begin{array}{l}\text { Iron } \\
\text { supplementation }\end{array}$ & 9 & $\begin{array}{l}\text { 1,254 apparently healthy } \\
\text { children }<5 \text { years of age }\end{array}$ & $\begin{array}{l}\text { Intermittent iron supplements } \\
\text { verses placebo }\end{array}$ & $\begin{array}{l}\mathrm{MD}(\mathrm{IV}, \mathrm{RE}, 95 \% \mathrm{Cl})= \\
6.45(2.36,10.55) \mathrm{g} / \mathrm{L}\end{array}$ & NR \\
\hline Eichler et al. (36) & $\begin{array}{l}\text { Iron + MMN } \\
\text { fortification }\end{array}$ & 8 & $\begin{array}{l}1,803 \text { Infants and children } \\
\text { from } 6 \text { months to } 5 \text { years } \\
\text { of age }\end{array}$ & $\begin{array}{l}\text { Iron + MMN fortification } \\
\text { verses non-fortified food }\end{array}$ & $\begin{array}{l}\mathrm{MD}(95 \% \mathrm{Cl})=0.87 \\
(0.57-1.16) \mathrm{g} / \mathrm{dL} \\
P=0.00 \\
R^{2}=81.5 \%\end{array}$ & NR \\
\hline Eichler et al. (36) & Iron fortification & 13 & $\begin{array}{l}2,274 \text { Infants and children } \\
\text { from } 6 \text { months to } 5 \text { years } \\
\text { of age }\end{array}$ & $\begin{array}{l}\text { Iron fortification of milk and } \\
\text { cereals verses non-fortified } \\
\text { food }\end{array}$ & $\begin{array}{l}\mathrm{MD}(95 \% \mathrm{Cl})=0.62 \\
(0.34,0.89) \mathrm{g} / \mathrm{dL} \\
P=0.00 \\
R^{2}=86.2 \%\end{array}$ & $N R$ \\
\hline $\begin{array}{l}\text { Gera et al. (44) } \\
\text { (change in } \mathrm{Hb} \text { ) }\end{array}$ & $\begin{array}{l}\text { Iron } \\
\text { supplementation/ } \\
\text { fortification }\end{array}$ & 44 & Children $\leq 5$ years of age & $\begin{array}{l}\text { Iron supplementation/ } \\
\text { fortification verses control }\end{array}$ & $\begin{array}{l}\text { WMD }(\mathrm{RE}, 95 \% \mathrm{Cl})= \\
0.59(0.43,0.75) \mathrm{g} / \mathrm{dL} \\
P<0.001\end{array}$ & $N R$ \\
\hline
\end{tabular}


TABLE 3 | Continued

\begin{tabular}{|c|c|c|c|c|c|c|}
\hline SR & Intervention & $\begin{array}{l}\text { \#Included } \\
\text { studies }\end{array}$ & \#Participants & Comparison & Meta-analysis & $\begin{array}{l}\text { Quality of } \\
\text { evidence }\end{array}$ \\
\hline $\begin{array}{l}\text { Cembranel et al. } \\
\text { (23) }\end{array}$ & $\begin{array}{l}\text { Iron } \\
\text { supplementation }\end{array}$ & 14 & Children $<5$ years of age & $\begin{array}{l}\text { Daily doses of FS verses } \\
\text { control }\end{array}$ & $\begin{array}{l}\mathrm{MD}(95 \% \mathrm{Cl})=0.56 \\
(0.31,0.81) \mathrm{mg} / \mathrm{dL} \\
P<0.001\end{array}$ & NR \\
\hline $\begin{array}{l}\text { Kristjansson et al. } \\
(41) \text { [change in } \mathrm{Hb} \\
(\mathrm{g} / \mathrm{L})]\end{array}$ & $\begin{array}{l}\text { Supplementary } \\
\text { feeding }\end{array}$ & 5 & $\begin{array}{l}300 \text { children aged } 3 \\
\text { months to } 5 \text { years in } \\
\text { LMICs }\end{array}$ & $\begin{array}{l}\text { Supplementary feeding } \\
\text { verses control }\end{array}$ & $\begin{array}{l}\text { SMD }(I V, R E, 95 \% C l)= \\
0.49(0.07-0.91) \mathrm{g} / \mathrm{L} \\
R^{2}=63 \%\end{array}$ & $N R$ \\
\hline $\begin{array}{l}\text { Matsuyama et al. } \\
(30)\end{array}$ & Milk fortification & NR & $\begin{array}{l}\text { Healthy children aged } \\
6-47 \text { months }\end{array}$ & Fortified milk verses control & $\begin{array}{l}\mathrm{MD}(95 \% \mathrm{Cl})=5.89 \\
(-0.24,12.02) \mathrm{g} / \mathrm{L} \\
P=0.06\end{array}$ & $N R$ \\
\hline $\begin{array}{l}\text { De-Regil et al. (31) } \\
\text { (g/L) }\end{array}$ & MNP fortification & 7 & $\begin{array}{l}\text { 2,023 children between } 2 \\
\text { and } 5 \text { years of age }\end{array}$ & $\begin{array}{l}\text { Point-of-use fortification of } \\
\text { foods with MNP verses no } \\
\text { intervention or placebo }\end{array}$ & $\begin{array}{l}\mathrm{MD}(\mathrm{IV}, \mathrm{RE}, 95 \% \mathrm{Cl})= \\
2.02(-0.87,4.92) \mathrm{g} / \mathrm{L}\end{array}$ & NR \\
\hline Eichler et al. (36) & Iron fortification & 5 & $\begin{array}{l}471 \text { Infants and children } \\
\text { from } 6 \text { months to } 5 \text { years } \\
\text { of age }\end{array}$ & $\begin{array}{l}\text { Iron single-fortification verses } \\
\text { non-fortified food }\end{array}$ & $\begin{array}{l}\mathrm{MD}(95 \% \mathrm{Cl})=0.20 \\
(-0.05-0.45) \mathrm{g} / \mathrm{dL} \\
P=0.132 \\
R^{2}=43.4 \%\end{array}$ & NR \\
\hline $\begin{array}{l}\text { Cembranel et al. } \\
\text { (23) }\end{array}$ & $\begin{array}{l}\text { Iron } \\
\text { supplementation }\end{array}$ & 07 & Children $<5$ years of age & $\begin{array}{l}\text { Weekly doses of FS verses } \\
\text { control }\end{array}$ & $\begin{array}{l}\mathrm{MD}(95 \% \mathrm{Cl})=0.28 \\
(-0.22,0.78) \mathrm{mg} / \mathrm{dL} \\
P=0.273\end{array}$ & NR \\
\hline $\begin{array}{l}\text { Cembranel et al. } \\
\text { (23) }\end{array}$ & $\begin{array}{l}\text { Iron } \\
\text { supplementation }\end{array}$ & 03 & Children $<5$ years of age & $\begin{array}{l}\text { Daily doses verses control } \\
\text { weekly doses of FS }\end{array}$ & $\begin{array}{l}\mathrm{MD}(95 \% \mathrm{Cl})=0.28 \\
(-0.01,0.56) \mathrm{mg} / \mathrm{dL} \\
P=0.057\end{array}$ & NR \\
\hline De-Regil et al. (28) & $\begin{array}{l}\text { Iron } \\
\text { supplementation }\end{array}$ & 14 & $\begin{array}{l}2,270 \text { apparently healthy } \\
\text { children }<5 \text { years of age }\end{array}$ & $\begin{array}{l}\text { Intermittent iron supplements } \\
\text { verses daily iron supplements }\end{array}$ & $\begin{array}{l}\mathrm{MD}(\mathrm{IV}, \mathrm{RE}, 95 \% \mathrm{Cl})= \\
-0.75(-1.80 \\
0.29) \mathrm{g} / \mathrm{L}\end{array}$ & NR \\
\hline \multicolumn{7}{|c|}{ CHILDREN <12 YEARS } \\
\hline De-Regil et al. (31) & MNP Fortification & 11 & $\begin{array}{l}2,746 \text { preschool and } \\
\text { school-age children }\end{array}$ & $\begin{array}{l}\text { Point-of-use fortification of } \\
\text { foods with MNP verses no } \\
\text { intervention or placebo }\end{array}$ & $\begin{array}{l}\mathrm{MD}(95 \% \mathrm{Cl})=3.37 \\
(0.94,5.80) \mathrm{g} / \mathrm{L}\end{array}$ & Low \\
\hline Das et al. (34) & Iron fortification & 16 & $\begin{array}{l}\text { 3,832 preschool and } \\
\text { school-going children }\end{array}$ & $\begin{array}{l}\text { Iron fortification verses } \\
\text { unfortified foods/ regular diet }\end{array}$ & $\begin{array}{l}\operatorname{SMD}(95 \% \mathrm{Cl})=0.46 \\
(0.14,0.50)\end{array}$ & Moderate \\
\hline Das et al. (34) & MMN fortification & 7 & $\begin{array}{l}\text { 1,543 preschool and } \\
\text { school-going children }\end{array}$ & $\begin{array}{l}\text { MMN fortification verses } \\
\text { unfortified foods/ regular diet }\end{array}$ & $\begin{array}{l}\operatorname{SMD}(95 \% \mathrm{Cl})=0.45 \\
(0.12,0.79)\end{array}$ & Moderate \\
\hline $\begin{array}{l}\text { Mayo-Wilson et al. } \\
\text { (22) }\end{array}$ & $\begin{array}{l}\text { Zn } \\
\text { supplementation }\end{array}$ & 27 & $\begin{array}{l}6,024 \text { Children between } 6 \\
\text { months and } 12 \text { years of } \\
\text { age }\end{array}$ & $\begin{array}{l}\text { Zinc supplementation verses } \\
\text { no zinc supplementation }\end{array}$ & $\begin{array}{l}\operatorname{SMD}(95 \% \mathrm{Cl})=-0.05 \\
(-0.10,0.00) \\
P=0.002 \\
R^{2}=45 \%\end{array}$ & Low \\
\hline $\begin{array}{l}\text { Mayo-Wilson et al. } \\
\text { (22) }\end{array}$ & $\begin{array}{l}\mathrm{Zn}+\text { Iron } \\
\text { supplementation }\end{array}$ & 8 & $\begin{array}{l}\text { 1,341 Children between } 6 \\
\text { months and } 12 \text { years of } \\
\text { age }\end{array}$ & $\begin{array}{l}\text { Zinc with iron } \\
\text { supplementation verses zinc } \\
\text { alone }\end{array}$ & $\begin{array}{l}\operatorname{SMD}(95 \% \mathrm{Cl})=-0.23 \\
(-0.34,-0.12) \\
P<0.0001 \\
R^{2}=79 \%\end{array}$ & Low \\
\hline De-Regil et al. (28) & $\begin{array}{l}\text { Iron } \\
\text { supplementation }\end{array}$ & 19 & $\begin{array}{l}\text { 3,032 apparently healthy } \\
\text { children }<12 \text { years }\end{array}$ & $\begin{array}{l}\text { Intermittent iron } \\
\text { supplementation verses } \\
\text { placebo/no intervention }\end{array}$ & $\begin{array}{l}\mathrm{MD}(\mathrm{IV}, \mathrm{RE}, 95 \% \mathrm{Cl})= \\
5.20(2.51,7.88) \mathrm{g} / \mathrm{L} \\
P=0.00015 \\
R^{2}=93 \%\end{array}$ & Low \\
\hline De-Regil et al. (28) & $\begin{array}{l}\text { Iron } \\
\text { supplementation }\end{array}$ & 7 & $\begin{array}{l}\text { 1,616 apparently healthy } \\
\text { children }<12 \text { years }\end{array}$ & $\begin{array}{l}\text { Intermittent iron } \\
\text { supplementation (0-3 } \\
\text { months duration) verses } \\
\text { placebo/no intervention }\end{array}$ & $\begin{array}{l}\mathrm{MD}(\mathrm{IV}, \mathrm{RE}, 95 \% \mathrm{Cl})= \\
5.16(0.90,9.36) \mathrm{g} / \mathrm{L}\end{array}$ & NR \\
\hline De-Regil et al. (28) & $\begin{array}{l}\text { Iron } \\
\text { supplementation }\end{array}$ & 12 & $\begin{array}{l}\text { 1,416 apparently healthy } \\
\text { children }<12 \text { years }\end{array}$ & $\begin{array}{l}\text { Intermittent iron } \\
\text { supplementation (>3 months } \\
\text { duration) verses placebo/no } \\
\text { intervention }\end{array}$ & $\begin{array}{l}\mathrm{MD}(\mathrm{IV}, \mathrm{RE}, 95 \% \mathrm{Cl})= \\
5.13(2.82,7.51) \mathrm{g} / \mathrm{L}\end{array}$ & NR \\
\hline De-Regil et al. (28) & $\begin{array}{l}\text { Iron } \\
\text { supplementation }\end{array}$ & 11 & $\begin{array}{l}\text { 1,699 apparently healthy } \\
\text { children }<12 \text { years }\end{array}$ & $\begin{array}{l}\text { Intermittent iron only } \\
\text { supplementation verses } \\
\text { placebo/no intervention }\end{array}$ & $\begin{array}{l}\mathrm{MD}(\mathrm{IV}, \mathrm{RE}, 95 \% \mathrm{Cl})= \\
4.41(1.32,7.50) \mathrm{g} / \mathrm{L}\end{array}$ & NR \\
\hline De-Regil et al. (28) & $\begin{array}{l}\text { Iron } \\
\text { supplementation }\end{array}$ & 4 & $\begin{array}{l}756 \text { apparently healthy } \\
\text { children }<12 \text { years }\end{array}$ & $\begin{array}{l}\text { Intermittent iron folic acid } \\
\text { supplementation verses } \\
\text { placebo/no intervention }\end{array}$ & $\begin{array}{l}\mathrm{MD}(\mathrm{IV}, \mathrm{RE}, 95 \% \mathrm{Cl})= \\
3.36(1.51,5.21) \mathrm{g} / \mathrm{L}\end{array}$ & NR \\
\hline
\end{tabular}


TABLE 3 | Continued

\begin{tabular}{|c|c|c|c|c|c|c|}
\hline SR & Intervention & $\begin{array}{l}\text { \#Included } \\
\text { studies }\end{array}$ & \#Participants & Comparison & Meta-analysis & $\begin{array}{l}\text { Quality of } \\
\text { evidence }\end{array}$ \\
\hline De-Regil et al. (28) & $\begin{array}{l}\text { Iron +Zinc } \\
\text { supplementation }\end{array}$ & 1 & $\begin{array}{l}77 \text { apparently healthy } \\
\text { children }<12 \text { years }\end{array}$ & $\begin{array}{l}\text { Intermittent iron with zinc } \\
\text { supplementation verses } \\
\text { placebo/no intervention }\end{array}$ & $\begin{array}{l}\mathrm{MD}(\mathrm{IV}, \mathrm{RE}, 95 \% \mathrm{Cl})= \\
-1.60(-8.09,4.89) \\
\mathrm{g} / \mathrm{L}\end{array}$ & NR \\
\hline De-Regil et al. (28) & $\begin{array}{l}\text { Iron + Vit C } \\
\text { supplementation }\end{array}$ & 1 & $\begin{array}{l}50 \text { apparently healthy } \\
\text { children }<12 \text { years }\end{array}$ & $\begin{array}{l}\text { Intermittent iron with vitamin } \\
\text { C supplementation verses } \\
\text { placebo/no intervention }\end{array}$ & $\begin{array}{l}\mathrm{MD}(\mathrm{IV}, \mathrm{RE}, 95 \% \mathrm{Cl})= \\
20.70(17.51,23.89) \\
\mathrm{g} / \mathrm{L}\end{array}$ & NR \\
\hline De-Regil et al. (28) & $\begin{array}{l}\text { Iron +MMN } \\
\text { supplementation }\end{array}$ & 4 & $\begin{array}{l}450 \text { apparently healthy } \\
\text { children }<12 \text { years }\end{array}$ & $\begin{array}{l}\text { Intermittent iron with MMN } \\
\text { supplementation verses } \\
\text { placebo/no intervention }\end{array}$ & $\begin{array}{l}\mathrm{MD}(\mathrm{IV}, \mathrm{RE}, 95 \% \mathrm{Cl})= \\
5.47(0.32,10.61) \mathrm{g} / \mathrm{L}\end{array}$ & NR \\
\hline De-Regil et al. (28) & $\begin{array}{l}\text { Iron } \pm \text { MMN } \\
\text { supplementation }\end{array}$ & 19 & $\begin{array}{l}\text { 2,851 apparently healthy } \\
\text { children }<12 \text { years }\end{array}$ & $\begin{array}{l}\text { Intermittent iron supplements } \\
\pm \mathrm{MMN} \text { verses daily iron } \\
\text { supplements } \pm \mathrm{MMN}\end{array}$ & $\begin{array}{l}\mathrm{MD}(\mathrm{IV}, \mathrm{RE}, 95 \% \mathrm{Cl})= \\
-0.60(-1.54,0.35) \mathrm{g} / \mathrm{L} \\
P=0.22 \\
R^{2}=56 \%\end{array}$ & Low \\
\hline De-Regil et al. (28) & $\begin{array}{l}\text { Iron } \\
\text { supplementation }\end{array}$ & 11 & $\begin{array}{l}\text { 1,455 apparently healthy } \\
\text { children }<12 \text { years }\end{array}$ & $\begin{array}{l}\text { Intermittent iron supplements } \\
\text { verses daily iron supplements } \\
\text { (0-3 months duration) }\end{array}$ & $\begin{array}{l}\mathrm{MD}(\mathrm{IV}, \mathrm{RE}, 95 \% \mathrm{Cl})= \\
0.47(-0.91,1.84) \mathrm{g} / \mathrm{L}\end{array}$ & NR \\
\hline De-Regil et al. (28) & $\begin{array}{l}\text { Iron } \\
\text { supplementation }\end{array}$ & 8 & $\begin{array}{l}\text { 1,387 apparently healthy } \\
\text { children }<12 \text { years }\end{array}$ & $\begin{array}{l}\text { Intermittent iron supplements } \\
\text { verses daily iron supplements } \\
\text { (> } 3 \text { months duration) }\end{array}$ & $\begin{array}{l}\mathrm{MD}(\mathrm{IV}, \mathrm{RE}, 95 \% \mathrm{Cl})= \\
-1.14(-2.07,-0.22) \\
\mathrm{g} / \mathrm{L}\end{array}$ & NR \\
\hline De-Regil et al. (28) & $\begin{array}{l}\text { Iron } \\
\text { supplementation }\end{array}$ & 15 & $\begin{array}{l}2,144 \text { apparently healthy } \\
\text { children }<12 \text { years }\end{array}$ & $\begin{array}{l}\text { Intermittent iron supplements } \\
\text { verses daily iron } \\
\text { supplements (Iron alone) }\end{array}$ & $\begin{array}{l}\mathrm{MD}(\mathrm{IV}, \mathrm{RE}, 95 \% \mathrm{Cl})= \\
-0.51(-1.61,0.59) \\
\mathrm{g} / \mathrm{L}\end{array}$ & NR \\
\hline De-Regil et al. (28) & $\begin{array}{l}\text { Iron } \\
\text { supplementation }\end{array}$ & 2 & $\begin{array}{l}408 \text { apparently healthy } \\
\text { children }<12 \text { years }\end{array}$ & $\begin{array}{l}\text { Intermittent iron supplements } \\
\text { verses daily iron } \\
\text { supplements (With folic acid) }\end{array}$ & $\begin{array}{l}\mathrm{MD}(\mathrm{IV}, \mathrm{RE}, 95 \% \mathrm{Cl})= \\
-2.26(-4.30,-0.22) \\
\mathrm{g} / \mathrm{L}\end{array}$ & NR \\
\hline De-Regil et al. (28) & $\begin{array}{l}\text { Iron } \\
\text { supplementation }\end{array}$ & 3 & $\begin{array}{l}299 \text { apparently healthy } \\
\text { children }<12 \text { years of age }\end{array}$ & $\begin{array}{l}\text { Intermittent iron supplements } \\
\text { verses daily iron } \\
\text { supplements (With MMN) }\end{array}$ & $\begin{array}{l}\mathrm{MD}(\mathrm{IV}, \mathrm{RE}, 95 \% \mathrm{Cl})= \\
0.61(-2.04,3.26) \mathrm{g} / \mathrm{L}\end{array}$ & NR \\
\hline De-Regil et al. (28) & $\begin{array}{l}\text { Iron } \\
\text { supplementation }\end{array}$ & 10 & $\begin{array}{l}\text { 1,778 apparently healthy } \\
\text { children between } 5 \text { and } 12 \\
\text { years of age }\end{array}$ & $\begin{array}{l}\text { Intermittent iron supplements } \\
\text { verses placebo or no } \\
\text { intervention }\end{array}$ & $\begin{array}{l}\mathrm{MD}(\mathrm{IV}, \mathrm{RE}, 95 \% \mathrm{Cl})= \\
4.04(0.30,7.78) \mathrm{g} / \mathrm{L}\end{array}$ & NR \\
\hline De-Regil et al. (28) & $\begin{array}{l}\text { Iron } \\
\text { supplementation }\end{array}$ & 5 & $\begin{array}{l}581 \text { apparently healthy } \\
\text { children between } 5 \text { and } \\
12 \text { years of age }\end{array}$ & $\begin{array}{l}\text { Intermittent iron supplements } \\
\text { verses daily iron supplements }\end{array}$ & $\begin{array}{l}\mathrm{MD}(\mathrm{IV}, \mathrm{RE}, 95 \% \mathrm{Cl})= \\
-0.31(-2.59,1.97) \\
\mathrm{g} / \mathrm{L}\end{array}$ & $N R$ \\
\hline \multicolumn{7}{|c|}{ CHILDREN <18 YEARS OF AGE } \\
\hline $\begin{array}{l}\text { Girum et al. (47) } \\
\text { (change in } \mathrm{Hb} \text { ) }\end{array}$ & Deworming & 8 & $\begin{array}{l}10,05,239 \text { school children } \\
\text { from Asia and Africa }\end{array}$ & Deworming verses control & $\begin{array}{l}\text { WMD }(\mathrm{FE}, 95 \% \mathrm{Cl})= \\
1.62(1.01-2.25) \mathrm{g} / \mathrm{dL} \\
P=0.873 \\
R^{2}=0 \%\end{array}$ & NR \\
\hline Low et al. (24) & $\begin{array}{l}\text { Iron } \\
\text { supplementation }\end{array}$ & 28 & $\begin{array}{l}6,545 \\
\text { primary-school-aged } \\
\text { children between } 3.3 \text { and } \\
15 \text { years }\end{array}$ & $\begin{array}{l}\text { Iron supplementation verses } \\
\text { placebo/anti-helminthic } \\
\text { therapy/Zinc/multi-vitamin }\end{array}$ & $\begin{array}{l}\mathrm{MD}(95 \% \mathrm{Cl})=8.38 \\
(6.21-10.56) \mathrm{g} / \mathrm{L} \\
P<0.001 \\
R^{2}=97 \%\end{array}$ & NR \\
\hline $\begin{array}{l}\text { Low et al. (20) } \\
\text { (end of treatment) }\end{array}$ & $\begin{array}{l}\text { Iron } \\
\text { supplementation }\end{array}$ & 10 & $\begin{array}{l}3,220 \text { participants } \\
\text { between } 12 \text { and } 18 y e a r s\end{array}$ & $\begin{array}{l}\text { Daily oral iron } \\
\text { supplementation with or } \\
\text { without other vitamins (folic } \\
\text { acid or vitamin C)/verses } \\
\text { control/placebo }\end{array}$ & $\begin{array}{l}\mathrm{MD}(\mathrm{IV}, \mathrm{RE}, 95 \% \mathrm{Cl})= \\
6.99(3.85,10.13) \mathrm{g} / \mathrm{L} \\
P=0.00001 \\
R^{2}=95 \%\end{array}$ & NR for subgroup \\
\hline Aaron et al. (32) & MMN fortification & 8 & $\begin{array}{l}\text { 3,835 apparently healthy } \\
\text { children between } 5 \text { and } \\
18 \text { years of age }\end{array}$ & $\begin{array}{l}\text { MMN fortified beverages } \\
\text { verses non-fortified } \\
\text { beverages }\end{array}$ & $\begin{array}{l}2.79(1.19,4.33) \mathrm{g} / \mathrm{L} \\
P=0.004 \\
R^{2}=92 \%\end{array}$ & Moderate \\
\hline $\begin{array}{l}\text { Ramakrishnan } \\
\text { et al. (29) }\end{array}$ & Iron interventions & 16 & $\begin{array}{l}\text { 2,542 children between } 6 \\
\text { months and } 18 \text { years }\end{array}$ & $\begin{array}{l}\text { Iron interventions verses } \\
\text { control }\end{array}$ & $\begin{array}{l}\text { WMD }(95 \% \mathrm{Cl})=1.49 \\
(0.46,2.51)\end{array}$ & $N R$ \\
\hline $\begin{array}{l}\text { Gera et al. (44) } \\
\text { (change in } \mathrm{Hb} \text { ) }\end{array}$ & $\begin{array}{l}\text { Iron } \\
\text { supplementation/fc }\end{array}$ & $\begin{array}{l}91 \\
\text { ification }\end{array}$ & $\begin{array}{l}12,198 \text { children }<18 \\
\text { years of age }\end{array}$ & $\begin{array}{l}\text { Iron supplementation/ } \\
\text { fortification verses control }\end{array}$ & $\begin{array}{l}\text { WMD }(95 \% \mathrm{Cl})=0.74 \\
(0.61-0.87) \mathrm{g} / \mathrm{dL} \\
P<0.001\end{array}$ & NR \\
\hline $\begin{array}{l}\text { Gera et al. (44) } \\
\text { (change in } \mathrm{Hb} \text { ) }\end{array}$ & $\begin{array}{l}\text { Iron } \\
\text { supplementation }\end{array}$ & 82 & Children $<18$ years of age & $\begin{array}{l}\text { Iron supplementation verses } \\
\text { control }\end{array}$ & $\begin{array}{l}\text { WMD }(95 \% \mathrm{Cl})=0.79 \\
(0.65,0.94) \mathrm{g} / \mathrm{dL} \\
P<0.001\end{array}$ & NR \\
\hline
\end{tabular}


TABLE 3 | Continued

\begin{tabular}{|c|c|c|c|c|c|c|}
\hline SR & Intervention & $\begin{array}{l}\text { \#Included } \\
\text { studies }\end{array}$ & \#Participants & Comparison & Meta-analysis & $\begin{array}{l}\text { Quality of } \\
\text { evidence }\end{array}$ \\
\hline $\begin{array}{l}\text { Gera et al. (44) } \\
\text { (change in } \mathrm{Hb} \text { ) }\end{array}$ & Iron fortification & 9 & Children $<18$ years of age & $\begin{array}{l}\text { Iron fortification verses } \\
\text { control }\end{array}$ & $\begin{array}{l}\text { WMD }(\mathrm{RE}, 95 \% \mathrm{Cl})= \\
0.25(0.02,0.52) \mathrm{g} / \mathrm{dL} \\
P=0.065\end{array}$ & NR \\
\hline $\begin{array}{l}\text { Gera et al. (44) } \\
\text { (change in } \mathrm{Hb} \text { ) }\end{array}$ & $\begin{array}{l}\text { Iron } \\
\text { supplementation/ } \\
\text { fortification }\end{array}$ & 47 & Children $>5$ years of age & $\begin{array}{l}\text { Iron supplementation/ } \\
\text { fortification verses control }\end{array}$ & $\begin{array}{l}\text { WMD }(\mathrm{RE}, 95 \% \mathrm{Cl})= \\
0.88(0.67,1.08) \mathrm{g} / \mathrm{dL} \\
P<0.001\end{array}$ & NR \\
\hline $\begin{array}{l}\text { Gera et al. (44) } \\
\text { (change in } \mathrm{Hb} \text { ) }\end{array}$ & $\begin{array}{l}\text { Iron } \\
\text { supplementation/for }\end{array}$ & $\begin{array}{l}13 \\
\text { ification }\end{array}$ & $\begin{array}{l}\text { Children }<18 \text { years of age } \\
\text { from developed countries }\end{array}$ & $\begin{array}{l}\text { Iron supplementation/ } \\
\text { fortification verses control }\end{array}$ & $\begin{array}{l}\text { WMD }(\mathrm{RE}, 95 \% \mathrm{Cl})= \\
0.46(0.13,0.78) \mathrm{g} / \mathrm{dL} \\
P=0.007\end{array}$ & $N R$ \\
\hline $\begin{array}{l}\text { Gera et al. (44) } \\
\text { (change in } \mathrm{Hb} \text { ) }\end{array}$ & $\begin{array}{l}\text { Iron } \\
\text { supplementation/ } \\
\text { fortification }\end{array}$ & 78 & $\begin{array}{l}\text { Children }<18 \text { years of age } \\
\text { from developing countries }\end{array}$ & $\begin{array}{l}\text { Iron supplementation/ } \\
\text { fortification verses control }\end{array}$ & $\begin{array}{l}\text { WMD }(\mathrm{RE}, 95 \% \mathrm{Cl})= \\
0.78(0.64,0.93) \mathrm{g} / \mathrm{dL} \\
P<0.001\end{array}$ & NR \\
\hline Das et al. (34) & $\begin{array}{l}\text { Vitamin A } \\
\text { fortification }\end{array}$ & 2 & $\begin{array}{l}1,538 \text { children }<18 \text { years } \\
\text { of age }\end{array}$ & $\begin{array}{l}\text { Vitamin A fortification verses } \\
\text { unfortified foods/ regular diet }\end{array}$ & $\begin{array}{l}\operatorname{SMD}(95 \% \mathrm{Cl})=0.48 \\
(0.07,0.89)\end{array}$ & Low \\
\hline Das et al. (34) & Iron fortification & 20 & $\begin{array}{l}\text { 4,176 children from } \\
\text { UMHICs }\end{array}$ & $\begin{array}{l}\text { Iron fortification verses } \\
\text { unfortified foods/ regular diet }\end{array}$ & $\begin{array}{l}\operatorname{SMD}(95 \% \mathrm{Cl})=0.67 \\
(0.36,0.97)\end{array}$ & Moderate \\
\hline Das et al. (34) & MMN fortification & 8 & 1,769 children from LMICs & $\begin{array}{l}\text { MMN fortification verses } \\
\text { unfortified foods/ regular diet }\end{array}$ & $\begin{array}{l}\operatorname{SMD}(95 \% \mathrm{Cl})=0.50 \\
(0.21,0.78)\end{array}$ & Moderate \\
\hline Das et al. (34) & MMN fortification & 6 & $\begin{array}{l}\text { 1,282 children from } \\
\text { UMHICs }\end{array}$ & $\begin{array}{l}\text { MMN fortification verses } \\
\text { unfortified foods/ regular diet }\end{array}$ & $\begin{array}{l}\operatorname{SMD}(95 \% \mathrm{Cl})=1.25 \\
(0.45,2.06)\end{array}$ & Moderate \\
\hline Das et al. (34) & Zinc fortification & NR & Children below 18 years & $\begin{array}{l}\text { Zinc fortification verses } \\
\text { unfortified foods/ regular diet }\end{array}$ & $\begin{array}{l}\operatorname{SMD}(95 \% \mathrm{Cl})=-0.11 \\
(-0.52,0.31)\end{array}$ & Low \\
\hline $\begin{array}{l}\text { Gera et al. (43) } \\
\text { (change in } \mathrm{Hb} \text { ) }\end{array}$ & $\begin{array}{l}\text { Iron and MMN } \\
\text { supplementation }\end{array}$ & 25 & $\begin{array}{l}4,981 \text { children }<15 \text { years } \\
\text { from developing countries }\end{array}$ & $\begin{array}{l}\text { Iron and MMN } \\
\text { supplementation verses } \\
\text { placebo }\end{array}$ & $\begin{array}{l}\text { WMD }(\mathrm{RE}, 95 \% \mathrm{Cl})= \\
0.65(0.50,0.80) \mathrm{g} / \mathrm{L} \\
P<0.001 \\
R^{2}=89.6 \%\end{array}$ & NR \\
\hline $\begin{array}{l}\text { Gera et al. (43) } \\
\text { (change in } \mathrm{Hb} \text { ) }\end{array}$ & $\begin{array}{l}\text { Iron and MMN } \\
\text { supplementation }\end{array}$ & 13 & $\begin{array}{l}1,483 \text { children }<15 \text { years } \\
\text { from developing countries }\end{array}$ & $\begin{array}{l}\text { Iron and MMN } \\
\text { supplementation verses iron } \\
\text { supplementation alone }\end{array}$ & $\begin{array}{l}\text { WMD }(95 \% \mathrm{Cl})=0.14 \\
(0.00,0.28) \mathrm{g} / \mathrm{L} \\
P=0.044 \\
R^{2}=76.0 \%\end{array}$ & $N R$ \\
\hline Gera et al. (43) & $\begin{array}{l}\text { Iron and } \\
\text { micronutrient } \\
\text { supplementation }\end{array}$ & 18 & $\begin{array}{l}\text { Children < } 15 \text { years from } \\
\text { developing countries }\end{array}$ & $\begin{array}{l}\text { Iron and micronutrient } \\
\text { supplementation verses } \\
\text { placebo }\end{array}$ & $\begin{array}{l}\text { WMD }(\mathrm{RE}, 95 \% \mathrm{Cl})= \\
0.69(0.48,0.91) \mathrm{g} / \mathrm{L} \\
P<0.001\end{array}$ & $N R$ \\
\hline Gera et al. (43) & $\begin{array}{l}\text { Iron and } \\
\text { micronutrient } \\
\text { fortification }\end{array}$ & 15 & $\begin{array}{l}\text { Children }<15 \text { years from } \\
\text { developing countries }\end{array}$ & $\begin{array}{l}\text { Iron and micronutrient } \\
\text { fortification verses placebo }\end{array}$ & $\begin{array}{l}\text { WMD }(\mathrm{RE}, 95 \% \mathrm{Cl})= \\
0.61(0.40,0.81) \mathrm{g} / \mathrm{L} \\
P<0.001\end{array}$ & $N R$ \\
\hline Gera et al. (43) & $\begin{array}{l}\text { Iron and } \\
\text { micronutrient } \\
\text { supplementation }\end{array}$ & 9 & $\begin{array}{l}\text { Children }<15 \text { years from } \\
\text { developing countries }\end{array}$ & $\begin{array}{l}\text { Iron and micronutrient } \\
\text { supplementation verses iron }\end{array}$ & $\begin{array}{l}\text { WMD }(95 \% \mathrm{Cl})=0.27 \\
(0.13,0.41) \mathrm{g} / \mathrm{L} \\
P<0.001 \\
R^{2}=8.5 \%\end{array}$ & $N R$ \\
\hline Gera et al. (43) & $\begin{array}{l}\text { Iron and } \\
\text { micronutrient } \\
\text { fortification }\end{array}$ & 6 & $\begin{array}{l}\text { Children }<15 \text { years from } \\
\text { developing countries }\end{array}$ & $\begin{array}{l}\text { Iron and micronutrient } \\
\text { fortification verses iron }\end{array}$ & $\begin{array}{l}\text { WMD }(95 \% \mathrm{Cl})=0.06 \\
\begin{array}{l}(20.15,0.27) \mathrm{g} / \mathrm{L} \\
P=0.55 \\
R^{2}=89.3 \%\end{array}\end{array}$ & $N R$ \\
\hline De-Regil et al. (31) & MNP Fortification & 3 & $\begin{array}{l}524 \text { children aged } \geq 5 \\
\text { years }\end{array}$ & $\begin{array}{l}\text { Point-of-use fortification of } \\
\text { foods with MNP verses no } \\
\text { intervention or placebo }\end{array}$ & $\begin{array}{l}\mathrm{MD}(\mathrm{IV}, \mathrm{RE}, 95 \% \mathrm{Cl})= \\
7.86(-0.76,16.49) \mathrm{g} / \mathrm{L}\end{array}$ & $N R$ \\
\hline $\begin{array}{l}\text { Neuberger et al. } \\
(21) \text { (end of } \\
\text { treatment) }\end{array}$ & $\begin{array}{l}\text { Iron } \\
\text { supplementation }\end{array}$ & 16 & $\begin{array}{l}5,261 \text { Children }<18 \text { years } \\
\text { of age from malaria } \\
\text { endemic areas }\end{array}$ & $\begin{array}{l}\text { Iron vs. placebo or no } \\
\text { treatment }\end{array}$ & $\begin{array}{l}\mathrm{MD}(\mathrm{IV}, \mathrm{RE}, 95 \% \mathrm{Cl})= \\
0.75(0.48,1.01) \mathrm{g} / \mathrm{dL} \\
P<0.00001 \\
R^{2}=93 \%\end{array}$ & $N R$ \\
\hline $\begin{array}{l}\text { Neuberger et al. } \\
\text { (21) (change in } \mathrm{Hb} \text { ) }\end{array}$ & $\begin{array}{l}\text { Iron } \\
\text { supplementation }\end{array}$ & 12 & $\begin{array}{l}2,462 \text { children }<18 \text { years } \\
\text { of age from malaria } \\
\text { endemic areas }\end{array}$ & $\begin{array}{l}\text { Iron vs. placebo or no } \\
\text { treatment }\end{array}$ & $\begin{array}{l}\mathrm{MD}(\mathrm{IV}, \mathrm{RE}, 95 \% \mathrm{Cl})= \\
0.67(0.42,0.92) \mathrm{g} / \mathrm{dL} \\
P<0.00001 \\
R^{2}=82 \%\end{array}$ & $N R$ \\
\hline $\begin{array}{l}\text { Neuberger et al. } \\
\text { (21) (end of } \\
\text { treatment) }\end{array}$ & $\begin{array}{l}\text { Iron plus folic acid } \\
\text { supplementation }\end{array}$ & 1 & $\begin{array}{l}124 \text { children }<18 \text { years of } \\
\text { age from malaria endemic } \\
\text { areas }\end{array}$ & $\begin{array}{l}\text { Iron plus folic acid vs. } \\
\text { placebo }\end{array}$ & $\begin{array}{l}\mathrm{MD}(\mathrm{IV}, \mathrm{RE}, 95 \% \mathrm{Cl})= \\
0.90(0.51,1.29) \mathrm{g} / \mathrm{dL} \\
P<0.00001 \\
R^{2}=\mathrm{NA}\end{array}$ & $N R$ \\
\hline
\end{tabular}


TABLE 3 | Continued

\begin{tabular}{|c|c|c|c|c|c|c|}
\hline SR & Intervention & $\begin{array}{l}\text { \#Included } \\
\text { studies }\end{array}$ & \#Participants & Comparison & Meta-analysis & $\begin{array}{l}\text { Quality of } \\
\text { evidence }\end{array}$ \\
\hline $\begin{array}{l}\text { Neuberger et al. } \\
\text { (21) (end of } \\
\text { treatment) }\end{array}$ & $\begin{array}{l}\text { Iron plus } \\
\text { anti-malarial } \\
\text { treatment }\end{array}$ & 1 & $\begin{array}{l}151 \text { children }<18 \text { years of } \\
\text { age from malaria endemic } \\
\text { areas }\end{array}$ & $\begin{array}{l}\text { Iron plus anti-malarial } \\
\text { treatment vs. placebo }\end{array}$ & $\begin{array}{l}\mathrm{MD}(\mathrm{IV}, \mathrm{RE}, 95 \% \mathrm{Cl})= \\
0.91(0.47,1.35) \mathrm{g} / \mathrm{dL} \\
R^{2}=\mathrm{NA}\end{array}$ & NR \\
\hline $\begin{array}{l}\text { Athuman et al. (50) } \\
\text { (mean change in } \\
\mathrm{Hb} \text { ) }\end{array}$ & $\begin{array}{l}\text { Anti-malarial } \\
\text { treatment }\end{array}$ & 4 & $\begin{array}{l}1,672 \text { children with } \\
\text { anemia in } \\
\text { Malaria-endemic areas }\end{array}$ & $\begin{array}{l}\text { Intermittent preventive } \\
\text { antimalarial treatment verses } \\
\text { placebo (IPT } \pm \text { iron and folic } \\
\text { acid verses placebo } \pm \text { iron } \\
\text { and folic acid) }\end{array}$ & $\begin{array}{l}\mathrm{MD}(\mathrm{IV}, \mathrm{FE})=0.32 \\
(0.19,0.45) \mathrm{g} / \mathrm{dL} \\
R^{2}=18 \%\end{array}$ & Low \\
\hline $\begin{array}{l}\text { Athuman et al. (50) } \\
\text { (mean } \mathrm{Hb} \text { ) }\end{array}$ & $\begin{array}{l}\text { Anti-malarial } \\
\text { treatment }\end{array}$ & 4 & $\begin{array}{l}1,672 \text { children with } \\
\text { anemia in } \\
\text { Malaria-endemic areas }\end{array}$ & $\begin{array}{l}\text { Intermittent preventive } \\
\text { antimalarial treatment verses } \\
\text { placebo (IPT } \pm \text { iron and folic } \\
\text { acid verses placebo } \pm \text { iron } \\
\text { and folic acid) }\end{array}$ & $\begin{array}{l}\mathrm{MD}(\mathrm{IV}, \mathrm{FE})=0.35 \\
(0.06,0.64) \mathrm{g} / \mathrm{dL} \\
R^{2}=76 \%\end{array}$ & Low \\
\hline $\begin{array}{l}\text { Taylor-Robinson } \\
\text { et al. (46) }\end{array}$ & Deworming & 4 & $\begin{array}{l}1,992 \text { children }<16 \text { years } \\
\text { in areas endemic for } \\
\text { intestinal helminthes }\end{array}$ & $\begin{array}{l}\text { Deworming drugs verses no } \\
\text { intervention }\end{array}$ & $\begin{array}{l}\mathrm{MD}(\mathrm{IV}, \mathrm{FE}, 95 \% \mathrm{Cl})= \\
0.02(-0.05,0.09) \\
\mathrm{g} / \mathrm{dL} \\
P>0.05\end{array}$ & Low \\
\hline $\begin{array}{l}\text { Taylor-Robinson } \\
\text { et al. (46) }\end{array}$ & Deworming & 2 & $\begin{array}{l}108 \text { children }<16 \text { years in } \\
\text { areas endemic for } \\
\text { intestinal helminthes or } \\
\text { children screened for } \\
\text { infection }\end{array}$ & $\begin{array}{l}\text { Single dose deworming } \\
\text { drugs verses no intervention }\end{array}$ & $\begin{array}{l}\mathrm{MD}(\mathrm{IV}, \mathrm{FE}, 95 \% \mathrm{Cl})= \\
0.37(0.10,0.64) \mathrm{g} / \mathrm{dL} \\
P=0.008 \\
R^{2}=0 \%\end{array}$ & Low \\
\hline $\begin{array}{l}\text { Taylor-Robinson } \\
\text { et al. (46) }\end{array}$ & Deworming & 2 & $\begin{array}{l}658 \text { children }<16 \text { years in } \\
\text { areas endemic for } \\
\text { intestinal helminthes } \\
\text { (moderate prevalence) }\end{array}$ & $\begin{array}{l}\text { Single dose deworming } \\
\text { drugs (targeted intervention) } \\
\text { verses no intervention }\end{array}$ & $\begin{array}{l}\mathrm{MD}(\mathrm{IV}, \mathrm{FE}, 95 \% \mathrm{Cl})= \\
0.06(-0.06,0.17) \\
\mathrm{g} / \mathrm{dL} \\
P=0.34 \\
P^{2}=0 \%\end{array}$ & $N R$ \\
\hline $\begin{array}{l}\text { Taylor-Robinson } \\
\text { et al. (46) }\end{array}$ & Deworming & 2 & $\begin{array}{l}1,334 \text { children }<16 \text { years } \\
\text { in areas endemic for } \\
\text { intestinal helminthes (low } \\
\text { prevalence) }\end{array}$ & $\begin{array}{l}\text { Single dose deworming } \\
\text { drugs (targeted intervention) } \\
\text { verses no intervention }\end{array}$ & $\begin{array}{l}\mathrm{MD}(\mathrm{IV}, \mathrm{FE}, 95 \% \mathrm{Cl})= \\
0.00(-0.08,0.08) \\
\mathrm{g} / \mathrm{dL} \\
P=0.92 \\
P^{2}=0 \%\end{array}$ & $N R$ \\
\hline $\begin{array}{l}\text { Taylor-Robinson } \\
\text { et al. (46) (in first } \\
\text { year) }\end{array}$ & Deworming & 4 & $\begin{array}{l}807 \text { children }<16 \text { years in } \\
\text { areas endemic for } \\
\text { intestinal helminthes }\end{array}$ & $\begin{array}{l}\text { Multiple dose deworming } \\
\text { drugs verses no intervention }\end{array}$ & $\begin{array}{l}\mathrm{MD}(\mathrm{IV}, \mathrm{FE}, 95 \% \mathrm{Cl})= \\
0.01(0.13,0.14) \mathrm{g} / \mathrm{dL}\end{array}$ & Low \\
\hline $\begin{array}{l}\text { Taylor-Robinson } \\
\text { et al. (46) (in first } \\
\text { year) }\end{array}$ & Deworming & 2 & $\begin{array}{l}464 \text { children }<16 \text { years in } \\
\text { areas endemic for } \\
\text { intestinal helminthes } \\
\text { (moderate prevalence) }\end{array}$ & $\begin{array}{l}\text { Multiple dose deworming } \\
\text { drugs (targeted intervention) } \\
\text { verses no intervention }\end{array}$ & $\begin{array}{l}\mathrm{MD}(\mathrm{IV}, \mathrm{FE}, 95 \% \mathrm{Cl})= \\
0.02(-0.15,0.19) \\
\mathrm{g} / \mathrm{dL} \\
P=0.82 \\
P^{2}=0 \%\end{array}$ & $N R$ \\
\hline $\begin{array}{l}\text { Taylor-Robinson } \\
\text { et al. (46) (in first } \\
\text { year) }\end{array}$ & Deworming & 2 & $\begin{array}{l}343 \text { children }<16 \text { years in } \\
\text { areas endemic for } \\
\text { intestinal helminthes (low } \\
\text { prevalence) }\end{array}$ & $\begin{array}{l}\text { Multiple dose deworming } \\
\text { drugs (targeted intervention) } \\
\text { verses no intervention }\end{array}$ & $\begin{array}{l}\mathrm{MD}(\mathrm{IV}, \mathrm{FE}, 95 \% \mathrm{Cl})= \\
-0.06(-0.28,0.17) \\
\mathrm{g} / \mathrm{dL} \\
P=0.62 \\
P^{2}=0 \%\end{array}$ & NR \\
\hline $\begin{array}{l}\text { Taylor-Robinson } \\
\text { et al. (46) (after first } \\
\text { year) }\end{array}$ & Deworming & 2 & $\begin{array}{l}1,365 \text { children }<16 \text { years } \\
\text { in areas endemic for } \\
\text { intestinal helminthes (low } \\
\text { prevalence) }\end{array}$ & $\begin{array}{l}\text { Multiple dose deworming } \\
\text { drugs (targeted intervention) } \\
\text { verses no intervention }\end{array}$ & $\begin{array}{l}\mathrm{MD}(\mathrm{IV}, \mathrm{FE}, 95 \% \mathrm{Cl})= \\
-0.02(-0.30,0.27) \\
\mathrm{g} / \mathrm{dL} \\
P=0.91 \\
R^{2}=0 \%\end{array}$ & Very low \\
\hline $\begin{array}{l}\text { Huang et al. (48) } \\
\text { (changes in } \mathrm{Hb} \text { ) }\end{array}$ & H. pylori treatment & 4 & $<18$ years of age & $\begin{array}{l}\text { H. pylori treatment for } \\
\text { eradication verses placebo }\end{array}$ & $\begin{array}{l}\text { WMD (IV, RE, 95\%CI) = } \\
\begin{array}{l}11.77(2.40,21.15) \mathrm{g} / \mathrm{L} \\
P=0.01 \\
R^{2}=90 \%\end{array}\end{array}$ & NR \\
\hline
\end{tabular}


-Das 2013b:Infants; MMN fortification* -Das 2013b:Infants; Iron fortification*

- Salam 2013: Infants; MMN supplimentation/fortification* -Pasricha 2013: Children 4M-23M; Iron Supplimentation* -De-Regil 2011b: Children 6M-23M; Home fortification with MMP* -Dewey 2009: Children 4M-2Y; Home fortification* -Dewey 2009: Children 6M-2Y; Home fortification (verses iron drops) De-Regil 2011: Children 6M-23M; Home fortification (verses iron -Thompson 2013: Children 2-5Y; Iron Supplimentation (daily)* $\rightarrow$ De-Regil 2011a: Children $<5 Y$; Iron Supplimentation* Eichler 2012: Children 6M-5Y; Iron+MMN Fortification*
. -Eichler 2012: Children $6 \mathrm{M}-5 \mathrm{Y}$; Iron fortification of milk and cereals * - Cembranel 2013: Children<5Y; FS Supplementation (daily)* - Kristjansson 2015: Children 3M-5Y; Supplementary feeding* - Matsuyama 2017: Children 6-47M; Milk Fortification* -De-Regil 2017: Children2-5Y; Point-of-use fortification of foods with MNP* $\rightarrow$ Eichler 2012: Children $6 \mathrm{M}-5 Y$; Single-iron fortification* $\rightarrow$-Cembranel 2013: Children<5Y; FS Supplementation (weekly)* -Cembranel 2013: Children<5Y; FS Supplementation (Daily doses verses weekly doses) -De-Regil 2011: Children<5Y; Iron Supplimentation (intermittent doses verses daily doses) -De Regil 2017: Pre-school \& school-going children; Point-of-use fortification with MNP* $\rightarrow$ Eichler 2012: Children 6M-5Y; Iron+MMN Fortification*

$\rightarrow-$ Das 2013b: Pre-school \& school-going children; Iron fortification

-Das 2013b. Pre-school \& school-going chidren, ron fortification *

- Das 2013 b. Pre-school \& school-going children, MMN fortification*

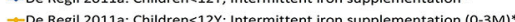

- De Regit 2011 a C Cirencis

-De Regil 2011a. Children<12Y; Intermitent iron supplementation (23M)*

DDe Regil 2011a: Children<12;; Intermittent iron (only) supple
-De Regil 2011a: Children<12Y; Iron+MMN supplementation*

-De Regil 2011a: Children<12Y; Iron+MMN supplementation (intermittent verses daily) De Regl 2011a: Chldrenk12y, iron MMN supplementation (intermittent verses daily) -De Regil 2011a: Cindrenk12y, hon supplementation (intermittent verses daily for 0-3M)

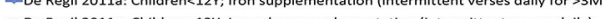

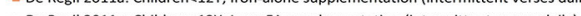
-De Regi 2011a. Children K12v, IrontFa supplementation (intermittent verses daily)

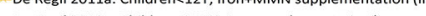
-De Regil 201la.Chidren 5 -12vifron supplementation

De Regil 2011a: Childen $5-12$; iron supplementation (intermittent verses daily)

-Low 2013. Chind - Low 2016: Children 12Y-18Y, iron supplementation wit/whout other vit (FA/Nit C)* Aaron 2015: Children 5Y-18Y; MMN fortification -Das 2013b: Children<18Y, Vit A fortification -Das 2013b: Childrenc18r, Iron fortification* Das 2013b: Children<18Y; MMN fortification De-Regil 2017: Children >/=5 Y; Point-of-use fortification with MNP* - Neuberger 2016: Children <18Y; Iron supplementation* (end of T/t) Neuberger 2016: Children <18Y in malaria endemic areas; Iron+Folic Acid supplementation* (end of $\mathrm{T} / \mathrm{t}$ ) Neuberger 2016: Children $<18 \mathrm{Y}$ in malaria endemic areas; Iron+anti-malarials*(end of $T / 4)$ Athuman 2015: Children <18Y; IPT*(Mean change in $\mathrm{Hb}$ )

- Taylor-Robbinson 2015: Children <16Y in areas endemic for intestinal helminths; Deworming

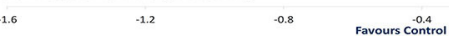

\section{Hemoglobin}

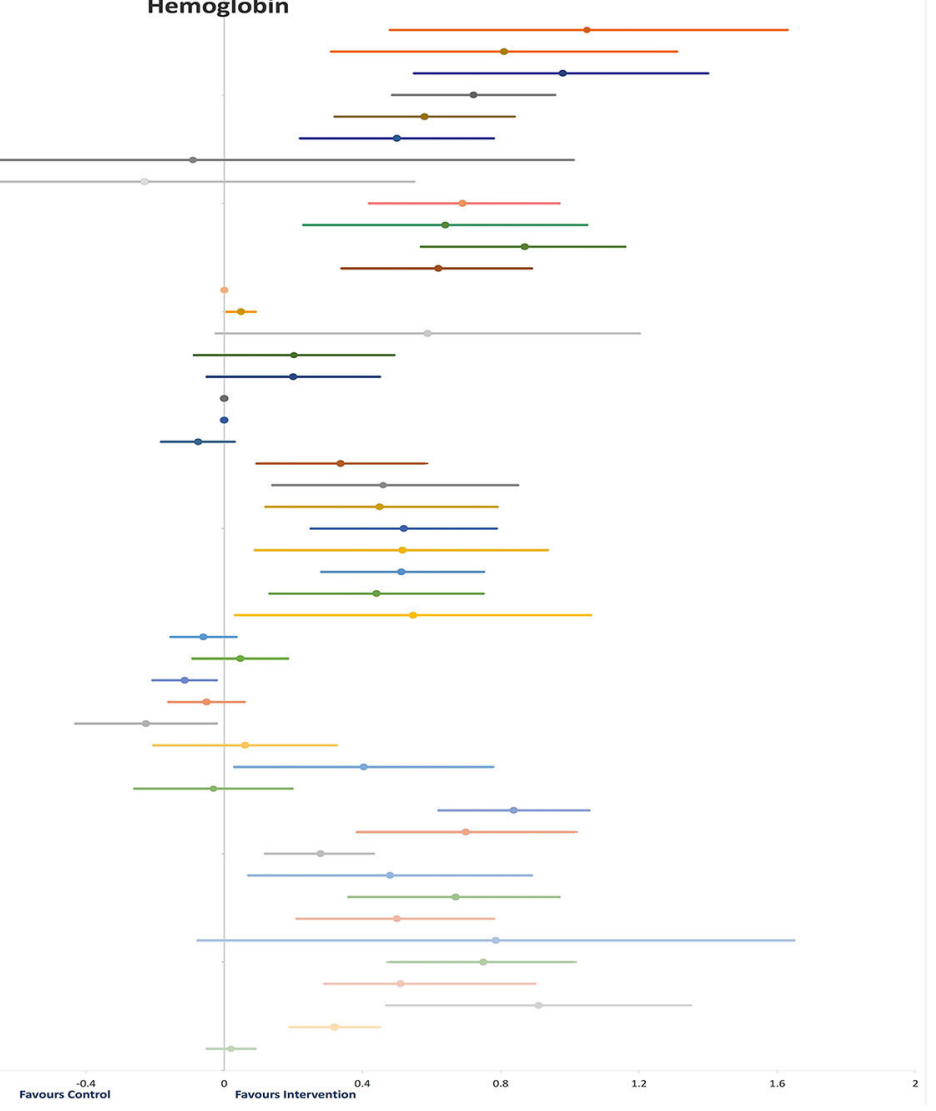

FIGURE 3 | Findings of SRs on $\mathrm{Hb}$.

(31). In apparently healthy children under 12 years of age, intermittent supplementation with iron alone or in combination with other nutrients effectively increased $\mathrm{Hb}$ concentrations, and this positive response did not differ between frequency or duration of supplementation or age of children (28).

In children under 15 years of age, daily iron supplementation $\left(\mathrm{MD}=8.38 \mathrm{~g} / \mathrm{L}, 95 \% \mathrm{CI}=6.21-10.56 ; I^{2}=97 \% ; 28\right.$ studies; $6,545$ participants $3.3-15 \mathrm{Y})(24)$ and iron with micronutrient supplementation $(\mathrm{WMD}=0.69 \mathrm{~g} / \mathrm{L}, 95 \% \mathrm{CI}=0.48-0.91 ; 18$ studies; participants $<15 \mathrm{Y}$ ) showed better improvements in $\mathrm{Hb}$ than iron with micronutrient fortification (WMD $=0.61$ $\mathrm{g} / \mathrm{L}, 95 \% \mathrm{CI}=0.40-0.81 ; 15$ studies; participants $<15 \mathrm{Y})(43)$. A school-based deworming program resulted in a decreased prevalence of anemia $\left(\mathrm{WMD}=1.62,95 \% \mathrm{CI}=1.01-2.25 ; I^{2}=\right.$ 0\%; eight studies; 1,005,239 participants) (47).

In children under 18 years of age, point-of-use fortification of food with MMP showed maximum though insignificant improvements in $\mathrm{Hb}$ levels $(\mathrm{MD}=7.86 \mathrm{~g} / \mathrm{L}, 95 \% \mathrm{CI}=-0.76-$ 16.49; three studies; 524 participants $\geq 5 \mathrm{Y}$ ) (31). Daily oral iron supplementation with or without other vitamins $(\mathrm{MD}=6.99 \mathrm{~g} / \mathrm{L}$, $95 \% \mathrm{CI}=3.85-10.13 ; I^{2}=95 \% ; 10$ studies; 3,220 participants 12-18Y) (20) and MMN-fortified beverages (2.76 g/L, 95\%CI =1.19-4.33; eight studies; 3,855 apparently healthy participants
5-18Y) showed significant improvements (32). Also, there was a substantially higher increase in $\mathrm{Hb}$ concentration from iron interventions $(\mathrm{WMD}=1.49,95 \% \mathrm{CI}=0.46-2.51 ; 16$ studies; $2,542$ participants $6 \mathrm{M}-18 \mathrm{Y})(29)$ than oral iron supplementation (WMD $=0.79 \mathrm{~g} / \mathrm{dL}, 95 \% \mathrm{CI}=0.65-0.94 ; 82$ studies $)$ and iron fortification $(\mathrm{WMD}=0.25 \mathrm{~g} / \mathrm{dL}, 95 \% \mathrm{CI}=0.02-0.52$; nine studies; participants <18Y) (44). Fortification of food with vitamin A substantially increased $\mathrm{Hb}$ concentrations in children under 18 years of age $(\mathrm{SMD}=0.48,95 \% \mathrm{CI}=0.07=0.89$; two studies; 1,538 participants $<18 \mathrm{Y})$, with iron $(\mathrm{SMD}=0.67,95 \% \mathrm{CI}$ $=0.36-0.97 ; 20$ studies; 4,176 participants $<18 \mathrm{Y}$ from UMHICs), and with $\mathrm{MMN}(\mathrm{SMD}=0.50,95 \% \mathrm{CI}=0.21-0.78$; eight studies; 1,769 participants $<18$ Y from LMICs) (34).

In children under 18 years of age residing in malaria-endemic areas, iron with antimalarial treatment $(\mathrm{MD}=0.91 \mathrm{~g} / \mathrm{dL}, 95 \% \mathrm{CI}$ $=0.47-1.35$; one study; 151 participants $<18 \mathrm{Y}$ ) and iron with FA supplementation $(\mathrm{MD}=0.90 \mathrm{~g} / \mathrm{dL}, 95 \% \mathrm{CI}=0.51-1.29$; one study; 124 participants $<18 \mathrm{Y}$ ) showed better results as compared with iron-only supplementation $(\mathrm{MD}=0.75 \mathrm{~g} / \mathrm{dL}, 95 \% \mathrm{CI}=$ $0.48-1.01 ; I^{2}=93 \% ; 16$ studies; 5,261 participants $\left.<18 \mathrm{Y}\right)(21)$. There is low-quality evidence that community deworming has little or no effect $(\mathrm{MD}=0.02 \mathrm{~g} / \mathrm{dL}, 95 \% \mathrm{CI}=-0.05$ to 0.09 ; $I^{2}=0 \%$; two studies; 464 participants $<16 \mathrm{Y}$ ) and that, in 
programs that treat only infected children, a single dose of deworming may improve $\mathrm{Hb}(\mathrm{MD}=0.37 \mathrm{~g} / \mathrm{dL}, 95 \% \mathrm{CI}=0.10-$ 0.64; two trials; 108 participants $<16$ Y; low QoE) (46). Additional H. pylori eradication therapy along with iron administration provided better improvements in iron deficiency anemia than iron administration alone $(\mathrm{WMD}=11.77 \mathrm{~g} / \mathrm{L}, 95 \% \mathrm{CI}=2.40-$ 21.15; $I^{2}=90 \%$; four studies; participants $\left.<18 \mathrm{Y}\right)(48)$.

\section{Adverse Events}

A total of six reviews addressed the adverse effects of different interventions for preventing, treating, and controlling anemia $(21,22,24,25,28,31)$ (Table 4). We did not find any review that assessed the adverse effects of antimalarial intervention, deworming, $H$. pylori treatment, or WASH interventions.

In children between 4 months and 2 years, there is an uncertain effect of risk of adverse events with iron supplements as compared with controls with a $10 \%$ increase in the risk of any adverse event $\left(\mathrm{RR}=1.10,95 \% \mathrm{CI}=0.98-1.25 ; I^{2}=0 \%\right.$; three studies, 912 participants), $38 \%$ increase in the risk of vomiting $\left(\mathrm{RR}=1.38,95 \% \mathrm{CI}=1.10-1.73 ; I^{2}=1 \%\right.$; three studies, 1,020 participants), and $3 \%$ increase in the risk of diarrhea $(R R=1.03$, 95\%CI $=0.86-1.23 ; I^{2}=0 \%$; six studies; 1,697 participants) (25).

In apparently healthy children under 12 years of age, intermittent supplementation of iron alone or with other nutrients showed a 3.87-times risk of any adverse events (RR $=3.87,95 \% \mathrm{CI}=0.19-76.92$; one study; 53 participants) (28). However, the risk of any adverse events was $40 \%$ less $(\mathrm{RR}=0.60$, $95 \% \mathrm{CI}=0.19-1.87 ; I^{2}=87 \%$; four studies; 895 participants), and the risk of diarrhea was 1.17 times higher $(\mathrm{RR}=1.17,95 \% \mathrm{CI}=$ $0.60-2.28 ; I^{2}=0 \%$; one study; 122 participants) with intermittent iron supplements with or without other micronutrients as compared with daily iron supplements with or without other micronutrients (28). There is an insignificant effect of food fortification on adverse effects $(\mathrm{RR}=1.09,95 \% \mathrm{CI}=0.16-7.42$; one study; 90 participants; moderate QoE) and diarrhea (low QoE) in preschool and school-age children (31). The risk of gastrointestinal upset was 1.3 times higher $(\mathrm{RR}=1.30,95 \% \mathrm{CI}=$ $0.89-1.91 ; I^{2}=0 \%$; four studies; 576 participants), constipation was 3.44 times higher $\left(\mathrm{RR}=3.44,95 \% \mathrm{CI}=0.66-19.68 ; I^{2}\right.$ $=6 \%$; two studies, 202 participants), and vomiting was $14 \%$ lower $\left(\mathrm{RR}=0.86,95 \% \mathrm{CI}=0.13-5.67 ; I^{2}=0 \%\right.$; two studies; 202 participants) with iron supplements in school-aged children between 3.3 and 15 years (24).

In children under 18 years old residing in malaria endemic areas, there is a significantly higher risk of diarrheal episodes per patient-month by $15 \%$ with iron supplements $(\mathrm{RR}=1.15,95 \% \mathrm{CI}$ $=1.06-1.26$; eight studies; 23,912 participants) and by $29 \%$ with Zn supplements $\left(\mathrm{RR}=1.29,95 \% \mathrm{CI}=1.15-1.44 ; I^{2}=30 \%\right.$; three studies, 6,346 participants) (21).

\section{DISCUSSION}

We evaluated 31 SRs that assessed the effects of different interventions for addressing anemia in children and adolescents. The highest number of SRs addressed food fortification and iron supplementation. None of the reviews were from India or included participants from India. Overall, the quality of the evidence reported by the SRs varied between moderate to low (Supplementary File 2).

In infants, iron fortification showed better results as compared with MMN fortification $(30,34)$. In children between 4 months and 2 years of age; home fortification, when used as a preventive measure, showed significant reduction in the risk of anemia followed by iron supplementation $(25,38,40)$. However, when used as a treatment measure, home fortification insignificantly increased the risk of anemia (40). This difference in the effect of home fortification when used as a preventive measure and as a treatment measure might be because the prevention trials were conducted on unselected populations, and the participants in the control group did not receive any treatment. On the contrary, the therapeutic trials were conducted on anemic subjects, and the participants in the control received medicinal iron drops (40).

In children under 5 years old and in school-age children, fortification of food with iron and MMN showed better improvement in $\mathrm{Hb}$ (moderate QoE) (34, 36). In apparently healthy children under 12 years old, intermittent supplementation of iron alone or in combination with other nutrients significantly increased $\mathrm{Hb}$ concentrations, and this positive response did not differ with frequency or duration of supplementation or age of children (28). The risk of anemia is higher with intermittent iron/FA/MMN supplementation as compared with daily iron/FA/MMN supplementation (28). A school-based deworming program resulted in a decrease in the prevalence of anemia in children (47). Programs that combined interventions, such as deworming with iron and nutrition supplementation, led to better effects as compared with single interventions (47). Daily oral iron supplementation with or without other vitamins $(20,24)$ and MMN-fortified beverages (32) showed maximum reduction in the risk of anemia in children between 3.5 and 18 years. In malaria endemic areas, iron supplementation with antimalarial treatment reduced the risk of anemia (21). There is low-quality evidence that community deworming has little or no effect (46).

In summary, results were favorable for iron with MMN fortification until school age and iron with $M M N$ supplementation in older children with a clear reduction in the risk of anemia and increase in $\mathrm{Hb}$ among the intervention groups of individual SRs. However, other interventions reported by SRs were inconclusive and all SRs suggested further research on the same.

The chances of developing anemia were less in children who received daily supplements as compared with those who received intermittent supplements. However, studies suggest that, in places and settings in which daily supplementation is not practical, intermittent supplementation can be used as a public health intervention to address childhood anemia. We did not find any review that assessed the effect of treatment of deworming, $H$. pylori infection, or WASH intervention on anemia status.

Limitations of the review: This overview examined the available evidence with respect to interventions addressing childhood and adolescent anemia without any restrictions on the type of interventions or language of publication. We did not restrict the search to Cochrane reviews, and inclusion of non-Cochrane reviews makes it more comprehensive. However, 
TABLE 4 | Findings for adverse events of different interventions for addressing childhood anemias in included systematic reviews.

\begin{tabular}{|c|c|c|c|c|c|c|}
\hline SR & & $\begin{array}{l}\text { \#Included } \\
\text { studies }\end{array}$ & \#Participants & Comparison & Meta-analysis & $\begin{array}{l}\text { Quality of } \\
\text { evidence }\end{array}$ \\
\hline \multicolumn{7}{|c|}{ CHILDREN <2 YEARS } \\
\hline $\begin{array}{l}\text { Pasricha et al. (25) } \\
\text { (vomiting) }\end{array}$ & Iron supplements & 3 & $\begin{array}{l}\text { 1,020 children } \\
\text { between } 4 \text { and } 23 \\
\text { months }\end{array}$ & $\begin{array}{l}\text { Iron supplementation } \\
\text { verses control }\end{array}$ & $\begin{array}{l}\mathrm{RR}(95 \% \mathrm{Cl})=1.38 \\
(1.10-1.73) \\
P=0.006 \\
R^{2}=1 \%\end{array}$ & NR \\
\hline $\begin{array}{l}\text { Pasricha et al. (25) } \\
\text { (diarrhea } \\
\text { prevalence) }\end{array}$ & Iron supplements & 6 & $\begin{array}{l}1,697 \text { children } \\
\text { between } 4 \text { and } 23 \\
\text { months }\end{array}$ & $\begin{array}{l}\text { Iron supplementation } \\
\text { verses control }\end{array}$ & $\begin{array}{l}\mathrm{RR}(95 \% \mathrm{Cl})=1.03 \\
(0.86-1.23) \\
P=0.78 \\
R^{2}=0 \%\end{array}$ & $N R$ \\
\hline $\begin{array}{l}\text { Pasricha et al. (25) } \\
\text { (constipation) }\end{array}$ & Iron supplements & 2 & $\begin{array}{l}570 \text { children between } \\
4 \text { and } 23 \text { months }\end{array}$ & $\begin{array}{l}\text { Iron supplementation } \\
\text { verses control }\end{array}$ & $\begin{array}{l}\mathrm{RR}(95 \% \mathrm{Cl})=0.54 \\
(0.05-5.83) \\
P=0.49 \\
R^{2}=77 \%\end{array}$ & NR \\
\hline $\begin{array}{l}\text { Pasricha et al. (25) } \\
\text { (any adverse } \\
\text { events) }\end{array}$ & Iron supplements & 3 & $\begin{array}{l}912 \text { children between } \\
4 \text { and } 23 \text { months }\end{array}$ & $\begin{array}{l}\text { Iron supplementation } \\
\text { verses control }\end{array}$ & $\begin{array}{l}\mathrm{RR}(95 \% \mathrm{Cl})=1.10 \\
(0.98-1.25) \\
P=0.12 \\
R^{2}=0 \%\end{array}$ & $N R$ \\
\hline \multicolumn{7}{|c|}{ CHILDREN <12 YEARS } \\
\hline De-Regil et al. (28) & Iron supplements & 1 & $\begin{array}{l}53 \text { apparently healthy } \\
\text { children }<5 \text { years of } \\
\text { age }\end{array}$ & $\begin{array}{l}\text { Intermittent } \\
\text { supplementation verses } \\
\text { placebo or no intervention }\end{array}$ & $\begin{array}{l}\mathrm{RR}(\mathrm{M}-\mathrm{H}, \mathrm{RE}, 95 \% \mathrm{Cl}) \\
=3.87(0.19,76.92)\end{array}$ & NR \\
\hline De-Regil et al. (28) & Iron supplements & 4 & $\begin{array}{l}895 \text { apparently healthy } \\
\text { children }<5 \text { years of } \\
\text { age }\end{array}$ & $\begin{array}{l}\text { Intermittent iron } \\
\text { supplements verses daily } \\
\text { iron supplements }\end{array}$ & $\begin{array}{l}\mathrm{RR}(\mathrm{M}-\mathrm{H}, \mathrm{RE}, 95 \% \mathrm{Cl}) \\
=0.60(0.19,1.87)\end{array}$ & NR \\
\hline De-Regil et al. (28) & Iron supplements & 1 & $\begin{array}{l}53 \text { apparently healthy } \\
\text { children between } 5 \\
\text { and } 12 \text { years of age }\end{array}$ & $\begin{array}{l}\text { Intermittent } \\
\text { supplementation verses } \\
\text { placebo or no intervention }\end{array}$ & $\begin{array}{l}\mathrm{RR}(\mathrm{M}-\mathrm{H}, \mathrm{RE}, 95 \% \mathrm{Cl}) \\
=3.87(0.19,76.92)\end{array}$ & NR \\
\hline $\begin{array}{l}\text { De-Regil et al. (28) } \\
\text { (any AE) }\end{array}$ & Iron supplements & 1 & $\begin{array}{l}53 \text { apparently healthy } \\
\text { children }<12 \text { years }\end{array}$ & $\begin{array}{l}\text { Intermittent } \\
\text { supplementation with iron } \\
\text { alone or with other } \\
\text { nutrients verses placebo or } \\
\text { no intervention }\end{array}$ & $\begin{array}{l}\mathrm{RR}(\mathrm{M}-\mathrm{H}, \mathrm{FE}, 95 \% \mathrm{Cl})= \\
3.87(0.19,76.92) \\
P=0.37 \\
R^{2}=\mathrm{NA}\end{array}$ & $N R$ \\
\hline $\begin{array}{l}\text { De-Regil et al. (28) } \\
\text { (nausea) }\end{array}$ & Iron supplements & 1 & $\begin{array}{l}64 \text { apparently healthy } \\
\text { children }<12 \text { years }\end{array}$ & $\begin{array}{l}\text { Intermittent } \\
\text { supplementation with iron } \\
\text { alone or with other } \\
\text { nutrients verses placebo or } \\
\text { no intervention }\end{array}$ & $\begin{array}{l}\mathrm{RR}(\mathrm{M}-\mathrm{H}, \mathrm{RE}, 95 \% \mathrm{Cl}) \\
2.82(0.12,66.82) \\
P=0.52 \\
R^{2}=\mathrm{NA}\end{array}$ & $N R$ \\
\hline $\begin{array}{l}\text { De-Regil et al. (28) } \\
\text { (any AE) }\end{array}$ & Iron supplements & 4 & $\begin{array}{l}895 \text { apparently healthy } \\
\text { children }<12 \text { years }\end{array}$ & $\begin{array}{l}\text { Intermittent iron } \\
\text { supplements } \pm \text { other } \\
\text { micronutrients verses daily } \\
\text { iron supplements } \pm \text { other } \\
\text { micronutrients }\end{array}$ & $\begin{array}{l}\mathrm{RR}(\mathrm{M}-\mathrm{H}, \mathrm{RE}, 95 \% \mathrm{Cl})= \\
0.60(0.19,1.87) \\
P=0.38 \\
R^{2}=87 \%\end{array}$ & NR \\
\hline $\begin{array}{l}\text { De-Regil et al. (28) } \\
\text { (diarrhea) }\end{array}$ & Iron supplements & 1 & $\begin{array}{l}122 \text { apparently healthy } \\
\text { children }<12 \text { years }\end{array}$ & $\begin{array}{l}\text { Intermittent iron } \\
\text { supplements } \pm \text { other } \\
\text { micronutrients verses daily } \\
\text { iron supplements } \pm \text { other } \\
\text { micronutrients }\end{array}$ & $\begin{array}{l}\mathrm{RR}(\mathrm{M}-\mathrm{H}, \mathrm{RE}, 95 \% \mathrm{Cl})= \\
1.17(0.60,2.28) \\
P=0.65 \\
R^{2}=\mathrm{NA}\end{array}$ & $N R$ \\
\hline $\begin{array}{l}\text { Mayo-Wilson et al. } \\
\text { (22) (incidence of } \\
\text { vomiting) }\end{array}$ & $\begin{array}{l}\mathrm{Zn} / \text { Iron } \\
\text { supplementation }\end{array}$ & 5 & $\begin{array}{l}\text { 4,095 Children } \\
\text { between } 6 \text { months } \\
\text { and } 12 \text { years of age }\end{array}$ & $\begin{array}{l}\text { Zinc supplementation } \\
\text { verses no zinc } \\
\text { supplementation }\end{array}$ & $\begin{array}{l}\mathrm{RR}(95 \% \mathrm{Cl})=1.68 \\
(1.61,1.75) \\
P<0.00001 \\
R^{2}=85 \%\end{array}$ & Low \\
\hline $\begin{array}{l}\text { Mayo-Wilson et al. } \\
\text { (22) (prevalence of } \\
\text { vomiting) }\end{array}$ & $\begin{array}{l}\mathrm{Zn} / \text { Iron } \\
\text { supplementation }\end{array}$ & 4 & $\begin{array}{l}\text { 35,192 Children } \\
\text { between } 6 \text { months } \\
\text { and } 12 \text { years of age }\end{array}$ & $\begin{array}{l}\text { Zinc supplementation } \\
\text { verses no zinc } \\
\text { supplementation }\end{array}$ & $\begin{array}{l}\mathrm{RR}(95 \% \mathrm{Cl})=1.29 \\
(1.14,1.46) \\
P=0.18 \\
R^{2}=37 \%\end{array}$ & Low \\
\hline $\begin{array}{l}\text { Mayo-Wilson et al. } \\
\text { (22) (study } \\
\text { withdrawal) }\end{array}$ & $\begin{array}{l}\mathrm{Zn} / \text { Iron } \\
\text { supplementation }\end{array}$ & 6 & $\begin{array}{l}\text { 4,263 Children } \\
\text { between } 6 \text { months } \\
\text { and } 12 \text { years of age }\end{array}$ & $\begin{array}{l}\text { Zinc supplementation } \\
\text { verses no zinc } \\
\text { supplementation }\end{array}$ & $\begin{array}{l}\operatorname{RR}(95 \% \mathrm{Cl})=1.75 \\
(0.93,3.32) \\
P=0.28 \\
R^{2}=21 \%\end{array}$ & Low \\
\hline
\end{tabular}


TABLE 4 | Continued

\begin{tabular}{|c|c|c|c|c|c|c|}
\hline SR & & $\begin{array}{l}\text { \#Included } \\
\text { studies }\end{array}$ & \#Participants & Comparison & Meta-analysis & $\begin{array}{l}\text { Quality of } \\
\text { evidence }\end{array}$ \\
\hline $\begin{array}{l}\text { Mayo-Wilson et al. } \\
\text { (22) (study } \\
\text { withdrawal) }\end{array}$ & $\begin{array}{l}\text { Zn/Iron } \\
\text { supplementation }\end{array}$ & 2 & $\begin{array}{l}557 \text { Children between } \\
6 \text { months and } 12 \\
\text { years of age }\end{array}$ & $\begin{array}{l}\text { Zinc with iron } \\
\text { supplementation verses } \\
\text { zinc alone supplementation }\end{array}$ & $\begin{array}{l}\mathrm{RR}(95 \% \mathrm{Cl})=1.41 \\
(0.91,2.18) \\
P=0.78 \\
R^{2}=0 \%\end{array}$ & Low \\
\hline $\begin{array}{l}\text { De-Regil et al. (31) } \\
\text { (adverse effects) }\end{array}$ & Food fortification & 1 & $\begin{array}{l}90 \text { Preschool and } \\
\text { school-age children }\end{array}$ & $\begin{array}{l}\text { Point-of-use fortification of } \\
\text { foods with MNP verses no } \\
\text { intervention or placebo }\end{array}$ & $\begin{array}{l}\mathrm{RR}(\mathrm{M}-\mathrm{H}, \mathrm{RE}, 95 \% \mathrm{Cl}) \\
=1.09(0.16-7.42) \\
P>0.05 \\
R^{2}=\mathrm{NA}\end{array}$ & Moderate \\
\hline $\begin{array}{l}\text { De-Regil et al. (31) } \\
\text { (diarrhea) }\end{array}$ & Food fortification & 2 & $\begin{array}{l}366 \text { Preschool and } \\
\text { school-age children }\end{array}$ & $\begin{array}{l}\text { Point-of-use fortification of } \\
\text { foods with MNP verses no } \\
\text { intervention or placebo }\end{array}$ & $\begin{array}{l}\mathrm{RR}(\mathrm{M}-\mathrm{H}, \mathrm{RE}, 95 \% \mathrm{Cl}) \\
=0.97(0.53,1.78) \\
P=0.93 \\
R^{2}=0 \%\end{array}$ & Low \\
\hline $\begin{array}{l}\text { Low et al. (24) } \\
\text { (gastrointestinal } \\
\text { upset) }\end{array}$ & $\begin{array}{l}\text { Iron } \\
\text { supplementation }\end{array}$ & 4 & $\begin{array}{l}576 \\
\text { primary-school-aged } \\
\text { children between } 3.3 \\
\text { and } 15 \text { years }\end{array}$ & $\begin{array}{l}\text { Iron supplementation } \\
\text { verses } \\
\text { placebo/antihelminthic } \\
\text { therapy/Zinc/multi-vitamin }\end{array}$ & $\begin{array}{l}\operatorname{RR}(95 \% \mathrm{Cl})=1.30 \\
(0.89-1.91) \\
P=0.2 \\
R^{2}=0 \%\end{array}$ & NR \\
\hline $\begin{array}{l}\text { Low et al. (24) } \\
\text { (constipation) }\end{array}$ & $\begin{array}{l}\text { Iron } \\
\text { supplementation }\end{array}$ & 2 & $\begin{array}{l}202 \\
\text { primary-school-aged } \\
\text { children between } 3.3 \\
\text { and } 15 \text { years }\end{array}$ & $\begin{array}{l}\text { Iron supplementation } \\
\text { verses } \\
\text { placebo/antihelminthic } \\
\text { therapy/Zinc/multi-vitamin }\end{array}$ & $\begin{array}{l}\operatorname{RR}(95 \% \mathrm{Cl})=3.44 \\
(0.66-19.68) \\
P=0.2 \\
R^{2}=6 \%\end{array}$ & $N R$ \\
\hline $\begin{array}{l}\text { Low et al. (24) } \\
\text { (vomiting) }\end{array}$ & $\begin{array}{l}\text { Iron } \\
\text { supplementation }\end{array}$ & 2 & $\begin{array}{l}202 \\
\text { primary-school-aged } \\
\text { children between } 3.3 \\
\text { and } 15 \text { years }\end{array}$ & $\begin{array}{l}\text { Iron supplementation } \\
\text { verses } \\
\text { placebo/antihelminthic } \\
\text { therapy/Zinc/multi-vitamin }\end{array}$ & $\begin{array}{l}\mathrm{RR}(95 \% \mathrm{Cl})=0.86 \\
(0.13-5.67) \\
P=0.9 \\
R^{2}=0 \%\end{array}$ & $N R$ \\
\hline $\begin{array}{l}\text { Neuberger et al. } \\
\text { (21) (diarrheal } \\
\text { episodes per } \\
\text { patient-month by } \\
\text { Zn supplements) }\end{array}$ & $\begin{array}{l}\text { Iron } \\
\text { supplementation }\end{array}$ & 8 & $\begin{array}{l}23,912 \text { children in } \\
\text { malaria endemic areas }\end{array}$ & $\begin{array}{l}\text { Iron vs. placebo or no } \\
\text { treatment }\end{array}$ & $\begin{array}{l}\mathrm{RR}(\mathrm{IV}, \mathrm{FE}, 95 \% \mathrm{Cl})= \\
1.15(1.06,1.26) \\
P=0.0014 \\
R^{2}=40 \%\end{array}$ & NR \\
\hline $\begin{array}{l}\text { Dewey et al. (40) } \\
\text { (diarrhea) }\end{array}$ & Food Fortification & 8 & $\begin{array}{l}808 \text { children between } \\
5.5 \text { and } 18 \text { years }\end{array}$ & $\begin{array}{l}\text { Treatment home } \\
\text { fortification verses control }\end{array}$ & $\begin{array}{l}-0.34(-0.78,0.03) \\
P=0.02\end{array}$ & NR \\
\hline $\begin{array}{l}\text { Dewey et al. (40) } \\
\text { (diarrhea) }\end{array}$ & Food Fortification & 10 & $\begin{array}{l}\text { 1,195 children } \\
\text { between } 5.5 \text { and } 18 \\
\text { years }\end{array}$ & $\begin{array}{l}\text { Preventive home } \\
\text { fortification verses control }\end{array}$ & $\begin{array}{l}\mathrm{RR}(95 \% \mathrm{Cl})=1.07 \\
(0.78,1.47) \\
P=0.72\end{array}$ & NR \\
\hline $\begin{array}{l}\text { Neuberger et al. } \\
\text { (21) (diarrheal } \\
\text { episodes per } \\
\text { patient-month } \\
\text { without Zn) }\end{array}$ & $\begin{array}{l}\text { Iron } \\
\text { supplementation }\end{array}$ & 7 & $\begin{array}{l}17,566 \text { children in } \\
\text { malaria endemic areas }\end{array}$ & $\begin{array}{l}\text { Iron vs. placebo or no } \\
\text { treatment }\end{array}$ & $\begin{array}{l}\mathrm{RR}(\mathrm{FE}, 95 \% \mathrm{Cl})=0.99 \\
(0.87,1.13) \\
P=0.93 \\
R^{2}=0 \%\end{array}$ & $N R$ \\
\hline $\begin{array}{l}\text { Neuberger et al. } \\
\text { (21) (diarrheal } \\
\text { episodes per } \\
\text { patient-month with } \\
\text { Zn) }\end{array}$ & $\begin{array}{l}\text { Iron } \\
\text { supplementation }\end{array}$ & 3 & $\begin{array}{l}6,346 \text { children in } \\
\text { malaria endemic areas }\end{array}$ & $\begin{array}{l}\text { Iron vs. placebo or no } \\
\text { treatment }\end{array}$ & $\begin{array}{l}\mathrm{RR}(\mathrm{IV}, \mathrm{FE}, 95 \% \mathrm{Cl})= \\
1.29(1.15,1.44) \\
P=0.000017 \\
R^{2}=30 \%\end{array}$ & NR \\
\hline
\end{tabular}

wide physiological variations during different stages of childhood could make the uniform application of interventions difficult. We acknowledge that not all SRs included in this overview were up to date. We found limited reviews on deworming, antimalarial interventions, $H$. pylori intervention, and WASH intervention, and hence, there is a need for primary studies in these areas. Some SRs did not consider socio-economic or nutritional status at baseline or did not report this clearly, which could have had an influence on some findings. In some reviews, there were too few data to reach a firm conclusion.

Strengths of the review: We estimate the potential bias in this overview as low. We followed the Cochrane handbook for methodology (19). The search was as comprehensive as possible. Screening of studies, data extraction, and assessing the methodological quality of reviews were done in duplicate. The authors of this review are from diverse disciplines (e.g., public health, biostatistics, nutrition, physiology, and maternal and child health), and this internal diversity may be an asset of this overview.

After an extensive literature search, we did not come across any overview of SRs that has addressed this area. This overview can help healthcare providers, consumers, public health specialists, and decision makers by providing a "bird's-eye view" of the efficacy of different public health interventions across reviews and age groups. 


\section{Implications of All the Available Evidence}

This overview identified interventions that had favorable effect sizes (which public health experts might contemplate using) as well as interventions that were mostly ineffective (which public health experts might want to restrict). More research is needed to assess the effect of deworming, antimalarial intervention, $H$. pylori treatment, and WASH intervention on anemia. Also, better recording of adverse events in primary studies is needed.

\section{CONCLUSION}

Results were favorable for iron and MMN fortification and supplementation with a clear reduction in the risk of anemia and increase in $\mathrm{Hb}$ levels across all age groups. Other interventions reported by the SRs were inconclusive and suggest further research. MMN improves outcomes better than single micronutrients. Also, better recording of adverse effects of different intervention is needed.

\section{DATA AVAILABILITY STATEMENT}

The original contributions presented in the study are included in the article/Supplementary Material, further inquiries can be directed to the corresponding author/s.

\section{REFERENCES}

1. King TC. Elsevier's Integrated Pathology. (2007). p. 263-89. Available online at: https://www.sciencedirect.com/science/article/pii/ B9780323043281500176 (accessed December 05, 2019).

2. WHO. The Global Prevalence of Anaemia in 2011. Geneva: World Health Organization (2015). Available online at: http://apps.who.int/iris/bitstream/ 10665/177094/1/9789241564960-eng.pdf?ua=1 (accessed December 05, 2019).

3. WHO. Global Health Estimates 2014 Summary Tables: Deaths by Cause, Age and Sex, by WHO Region, 2000-2012. Geneva: World Health Organization (2014). Available online at: http://www.who.int/healthinfo/global_burden_ disease/estimates/en/index1.html (accessed December 05, 2019).

4. Bharati S, Pal M, Chakrabarty S, Bharati P. Socioeconomic determinants of iron-deficiency anemia among children aged 6 to 59 months in India. Asia Pac J Public Health. (2015) 27:NP1432-43. doi: 10.1177/1010539513491417

5. Singh RK, Patra S. Extent of anaemia among preschool children in EAG states, India: a challenge to policy makers. Anemia. (2014) 2014: 868752. doi: $10.1155 / 2014 / 868752$

6. Kassebaum NJ, Jasrasaria R, Naghavi M, Wulf SK, Johns N, Lozano R, et al. A systematic analysis of global anemia burden from 1990 to 2010. Blood. (2014) 123, 615-24. doi: 10.1182/blood-2013-06-508325

7. Soliman A, Kalra S, Sanctis V. Anemia and growth. Indian J Endocrinol Metab. (2014) 18(Suppl. 1): S1-5. doi: 10.4103/2230-8210.145038

8. Joo EY, Kim KY, Kim DH, Lee J-E, Kim SK. Iron deficiency anemia in infants and toddlers. Blood Res. (2016) 51:268. doi: 10.5045/br.2016.51.4.268

9. Carter RC, Jacobson JL, Burden MJ, Armony-Sivan R, Dodge NC, Angelilli $\mathrm{ML}$, et al. Iron deficiency anemia and cognitive function in infancy. Pediatrics. (2010) 126:e427-34. doi: 10.1542/peds.2009-2097

10. Khedr E, Hamed SA, Elbeih E, El-Shereef H, Ahmad Y, Ahmed S. Iron states and cognitive abilities in young adults: neuropsychological and neurophysiological assessment. Eur Arch Psychiatry Clin Neurosci. (2008) 258:489-96. doi: 10.1007/s00406-008-0822-y

11. Lozoff B. Iron deficiency and child development. Food Nutr Bull. (2007) 28(Suppl. 4):S560-71. doi: 10.1177/15648265070284S409

\section{AUTHOR CONTRIBUTIONS}

AS envisioned the idea of the review. RV developed and ran the search strategies. AS and PM developed the protocol. PM and RH screened title and abstract. RH and NK screened full texts. BU resolved disagreement among primary reviewers (for screening) through discussion. AS, PM, and MK extracted data. AS and PM assessed the methodological quality of included SRs. NN, AG, and SQ provided technical advice. MK and $P M$ drafted the manuscript with input from AS, BU, NN, AG, and SQ. All authors have contributed significantly to this overview, read the manuscript, and participated in writing and revision.

\section{ACKNOWLEDGMENTS}

Dr. Penny Holding, Grand Challenge Canada.

\section{SUPPLEMENTARY MATERIAL}

The Supplementary Material for this article can be found online at: https://www.frontiersin.org/articles/10.3389/fped. 2020.549549/full\#supplementary-material

12. Lukusa Kazadi A, Ngiyulu RM, Gini-Ehungu JL, Mbuyi-Muamba JM, Aloni MN. Factors associated with growth retardation in children suffering from sickle cell anemia: first report from central Africa. Anemia. (2017) 2017:7916348. doi: 10.1155/2017/7916348

13. Baltussen R, Knai C, Sharan M. Iron fortification and iron supplementation are cost-effective interventions to reduce iron deficiency in four subregions of the world. J Nutr. (2004) 134:2678-84. doi: 10.1093/jn/134. 10.2678

14. Prasanna Mithra, Anju Sinha, Nithin Kumar, Ramesh Holla, Bhaskaran Unnikrishnan, Mahalaqua Nazli Khatib, et al. Interventions for the control and prevention of anaemia among children and adolescents: an overview of systematic reviews. PROSPERO. (2016) CRD42016053687.

15. Covidence Systematic Review Software. Melbourne, VIC: Veritas Health Innovation. Available online at: www.covidence.org (accessed December 5, 2019).

16. Liberati A, Altman DG, Tetzlaff J, Mulrow C, Gøtzsche PC, Ioannidis JPA, et al. The PRISMA statement for reporting systematic reviews and metaanalyses of studies that evaluate healthcare interventions: explanation and elaboration. BMJ. (2009) 339:b2700. doi: 10.1136/bmj.b2700

17. Shea BJ, Hamel C, Wells GA, Bouter LM, Kristjansson E, Grimshaw J, et al. AMSTAR is a reliable and valid measurement tool to assess the methodological quality of systematic reviews. J Clin Epidemiol. (2009) 62:1013-20. doi: 10.1016/j.jclinepi.2008.10.009

18. Shea BJ, Reeves BC, Wells G, Thuku M, Hamel C, Moran J, et al. AMSTAR 2: a critical appraisal tool for systematic reviews that include randomised or non-randomised studies of healthcare interventions, or both. BMJ. (2017) 358:j4008. doi: 10.1136/bmj.j4008

19. Higgins JPT, Green S. Cochrane Handbook for Systematic Reviews of Interventions. Vol. Version 5.1.0. The Cochrane Collaboration (2011). Available online at: http://handbook.cochrane.org (accessed January 02, 2019).

20. Low MSY, Speedy J, Styles CE, De-Regil LM, Pasricha S-R. Daily iron supplementation for improving anaemia, iron status and health in menstruating women. Cochrane Database Syst Rev. (2016) 4:CD009747. doi: 10.1002/14651858.CD009747.pub2 
21. Neuberger A, Okebe J, Yahav D, Paul M. Oral iron supplements for children in malaria-endemic areas. Cochrane Database Syst Rev. (2016) 2:CD006589. doi: 10.1002/14651858.CD006589.pub4

22. Mayo-Wilson E, Imdad A, Junior J, Dean S, Bhutta ZA. Preventive zinc supplementation for children, and the effect of additional iron: a systematic review and meta-analysis. BMJ Open. (2014) 4:e004647. doi: 10.1136/bmjopen-2013-004647

23. Cembranel F, Dallazen C, González-Chica DA. [Effectiveness of ferrous sulfate supplementation in the prevention of anemia in children: a systematic literature review and meta-analysis]. Cad Saude Publica. (2013) 29:1731-51. doi: 10.1590/S0102-311X2013001300014

24. Low M, Farrell A, Biggs B-A, Pasricha S-R. Effects of daily iron supplementation in primary-school-aged children: systematic review and meta-analysis of randomized controlled trials. CMAJ. (2013) 185:E791-802. doi: $10.1503 / \mathrm{cmaj} .130628$

25. Pasricha S-R, Hayes E, Kalumba K, Biggs B-A. Effect of daily iron supplementation on health in children aged 4-23 months: a systematic review and meta-analysis of randomised controlled trials. Lancet Glob Health. (2013) 1:e77-86. doi: 10.1016/S2214-109X(13)70046-9

26. Thompson J, Biggs B-A, Pasricha S-R. Effects of daily iron supplementation in 2- to 5-year-old children: systematic review and meta-analysis. Pediatrics. (2013) 131:739-53. doi: 10.1542/peds.2012-2256

27. Abdullah K, Kendzerska T, Shah P, Uleryk E, Parkin PC. Efficacy of oral iron therapy in improving the developmental outcome of pre-school children with non-anaemic iron deficiency: a systematic review. Public Health Nutr. (2013) 16:1497-506. doi: 10.1017/S1368980012003709

28. De-Regil LM, Jefferds MED, Sylvetsky AC, Dowswell T. Intermittent iron supplementation for improving nutrition and development in children under 12 years of age. Cochrane Database Syst Rev. (2011) 2011:CD009085. doi: 10.1002/14651858.CD009085.pub2

29. Ramakrishnan U, Aburto N, McCabe G, Martorell R. Multimicronutrient interventions but not vitamin a or iron interventions alone improve child growth: results of 3 meta-analyses. J Nutr. (2004) 134:2592-602. doi: $10.1093 / \mathrm{jn} / 134.10 .2592$

30. Matsuyama M, Harb T, David M, Davies PS, Hill RJ. Effect of fortified milk on growth and nutritional status in young children: a systematic review and meta-analysis. Public Health Nutr. (2017) 20:1214-25. doi: $10.1017 /$ S1368980016003189

31. De-Regil LM, Jefferds MED, Peña-Rosas JP. Point-of-use fortification of foods with micronutrient powders containing iron in children of preschool and school-age. Cochrane Database Syst Rev. (2017) 11:CD009666. doi: 10.1002/14651858.CD009666.pub2

32. Aaron GJ, Dror DK, Yang Z. Multiple-micronutrient fortified non-dairy beverage interventions reduce the risk of anemia and iron deficiency in school-aged children in low-middle income countries: a systematic review and meta-analysis (i-iv). Nutrients. (2015) 7:3847-68. doi: 10.3390/nu7053847

33. Das JK, Kumar R, Salam RA, Bhutta ZA. Systematic review of zinc fortification trials. Ann Nutr Metab. (2013) 62(Suppl. 1):44-56. doi: 10.1159/000348262

34. Das JK, Salam RA, Kumar R, Bhutta ZA. Micronutrient fortification of food and its impact on woman and child health: a systematic review. Syst Rev. (2013) 2:67. doi: 10.1186/2046-4053-2-67

35. Salam RA, MacPhail C, Das JK, Bhutta ZA. Effectiveness of Micronutrient Powders (MNP) in women and children. BMC Public Health. (2013) 13(Suppl. 3):S22. doi: 10.1186/1471-2458-13-S3-S22

36. Eichler K, Wieser S, Rüthemann I, Brügger U. Effects of micronutrient fortified milk and cereal food for infants and children: a systematic review. BMC Public Health. (2012) 12:506. doi: 10.1186/1471-2458-12-506

37. Gera T, Sachdev HS, Boy E. Effect of iron-fortified foods on hematologic and biological outcomes: systematic review of randomized controlled trials. Am J Clin Nutr. (2012) 96:309-24. doi: 10.3945/ajcn.111. 031500

38. De-Regil LM, Suchdev PS, Vist GE, Walleser S, Peña-Rosas JP. Home fortification of foods with multiple micronutrient powders for health and nutrition in children under two years of age. Cochrane Database Syst Rev. (2011) 6:CD008959. doi: 10.1002/14651858.CD008959.pub2

39. Best C, Neufingerl N, Del Rosso JM, Transler C, van den Briel T, Osendarp S. Can multi-micronutrient food fortification improve the micronutrient status, growth, health, and cognition of schoolchildren? A systematic review. Nutr Rev. (2011) 69:186-204. doi: 10.1111/j.1753-4887.2011.00378.x

40. Dewey KG, Yang Z, Boy E. Systematic review and meta-analysis of home fortification of complementary foods. Matern Child Nutr. (2009) 5:283-321. doi: 10.1111/j.1740-8709.2009.00190.x

41. Kristjansson E, Francis DK, Liberato S, Benkhalti Jandu M, Welch V, Batal $\mathrm{M}$, et al. Food supplementation for improving the physical and psychosocial health of socio-economically disadvantaged children aged three months to five years. Cochrane Database Syst Rev. (2015) 2015:CD009924. doi: 10.1002/14651858.CD009924.pub2

42. McDonagh MS, Blazina I, Dana T, Cantor A, Bougatsos C. Screening and routine supplementation for iron deficiency anemia: a systematic review. Pediatrics. (2015) 135:723-33. doi: 10.1542/peds.2014-3979

43. Gera T, Sachdev HPS, Nestel P. Effect of combining multiple micronutrients with iron supplementation on $\mathrm{Hb}$ response in children: systematic review of randomized controlled trials. Public Health Nutr. (2009) 12:756-73. doi: $10.1017 /$ S1368980008003145

44. Gera T, Sachdev HPS, Nestel P, Sachdev SS. Effect of iron supplementation on haemoglobin response in children: systematic review of randomised controlled trials. J Pediatr Gastroenterol Nutr. (2007) 44:468-86. doi: 10.1097/01.mpg.0000243440.85 452.38

45. Sun J, Zhang L, Cui J, Li S, Lu H, Zhang Y, et al. Effect of dietary intervention treatment on children with iron deficiency anemia in China: a meta-analysis. Lipids Health Dis. (2018) 17:108. doi: 10.1186/s12944-0180749-x

46. Taylor-Robinson DC, Maayan N, Soares-Weiser K, Donegan S, Garner P. Deworming drugs for soil-transmitted intestinal worms in children: effects on nutritional indicators, haemoglobin, and school performance. In: The Cochrane Collaboration, editor. Cochrane Database of Systematic Reviews. Chichester: John Wiley \& Sons, Ltd. (2015) 7. doi: 10.1002/14651858.CD000371.pub6

47. Girum T, Wasie A. The effect of deworming school children on anemia prevalence: a systematic review and meta-analysis. Open Nurs J. (2018) 12:155-61. doi: 10.2174/1874434601812010155

48. Huang X, Qu X, Yan W, Huang Y, Cai M, Hu B, et al. Iron deficiency anaemia can be improved after eradication of Helicobacter pylori. Postgrad Med J. (2010) 86:272-8. doi: 10.1136/pgmj.2009. 089987

49. Dangour AD, Watson L, Cumming O, Boisson S, Che Y, Velleman Y, et al. Interventions to improve water quality and supply, sanitation and hygiene practices, and their effects on the nutritional status of children. Cochrane Database Syst Rev. (2013) 8:CD009382. doi: 10.1002/14651858.CD009 382.pub2

50. Athuman M, Kabanywanyi AM, Rohwer AC. Intermittent preventive antimalarial treatment for children with anaemia. Cochrane Database Syst Rev. (2015) 1:CD010767. doi: 10.1002/14651858.CD01 0767.pub2

51. Ojukwu JU, Okebe JU, Yahav D, Paul M. Oral iron supplementation for preventing or treating anaemia among children in malariaendemic areas. Cochrane Database Syst Rev. (2009) 3:CD006589. doi: 10.1002/14651858.CD006589.pub2

52. Okebe JU, Yahav D, Shbita R, Paul M. Oral iron supplements for children in malaria-endemic areas. Cochrane Database Syst Rev. (2011) 10:CD006589. doi: 10.1002/14651858.CD006589.pub3

53. Dickson RC, Awasthi S, Demellweek C, Williamson PR. Anthelmintic drugs for treating worms in children: effects on growth and cognitive performance. Cochrane Database Syst Rev. (2007) 2:CD000371. doi: 10.1002/14651858.CD00 0371.pub2

54. Taylor-Robinson DC, Maayan N, Soares-Weiser K, Donegan S, Garner P. Deworming drugs for soil-transmitted intestinal worms in children: effects on nutritional indicators, haemoglobin and school performance. Cochrane Database Syst Rev. (2012) 7:CD000371. doi: 10.1002/14651858.CD000 371.pub4

55. Taylor-Robinson DC, Jones AP, Garner P. Deworming drugs for treating soil-transmitted intestinal worms in children: effects on growth and 
school performance. Cochrane Database Syst Rev. (2007) 4:CD000371. doi: 10.1002/14651858.CD000371.pub3

56. De-Regil LM, Suchdev PS, Vist GE, Walleser S, Peña-Rosas JP. Home fortification of foods with multiple micronutrient powders for health and nutrition in children under two years of age (review). Evid Based Child Health Cochrane Rev J. (2013) 8:112-201. doi: 10.1002/ ebch. 1895

57. Pachón H, Spohrer R, Mei Z, Serdula MK. Evidence of the effectiveness of flour fortification programs on iron status and anemia: a systematic review. Nutr Rev. (2015) 73:780-95. doi: 10.1093/nutrit/ nuv037
Conflict of Interest: The authors declare that the research was conducted in the absence of any commercial or financial relationships that could be construed as a potential conflict of interest.

Copyright $\odot 2021$ Mithra, Khatib, Sinha, Kumar, Holla, Unnikrishnan, Vijayamma, Nair, Gaidhane and Quazi Zahiruddin. This is an open-access article distributed under the terms of the Creative Commons Attribution License (CC BY). The use, distribution or reproduction in other forums is permitted, provided the original author(s) and the copyright owner(s) are credited and that the original publication in this journal is cited, in accordance with accepted academic practice. No use, distribution or reproduction is permitted which does not comply with these terms. 